\title{
Endoplasmic Reticulum Stress in Spinal Cord Contributes to the Development of Morphine Tolerance
}

\author{
Daiqiang Liu', Yaqun Zhou', Yawen Peng' ${ }^{2}$, Peng Su', Zheng Li ${ }^{1}$, Qiaoqiao Xu' ${ }^{1}$, Ye Tu ${ }^{1}$, \\ Xuebi Tian', Hui Yang ${ }^{1}$, Zhen Wu' ${ }^{1}$, Wei Mei ${ }^{1}$ and Feng Gao ${ }^{1 *}$ \\ ${ }^{1}$ Department of Anesthesiology, Tongji Hospital, Tongji Medical College, Huazhong University of Science and Technology, \\ Wuhan, China, ${ }^{2}$ Department of Anesthesiology, Renmin Hospital of Wuhan University, Hubei General Hospital, Wuhan, China
}

Morphine tolerance remains an intractable problem, which hinders its prolonged use in clinical practice. Endoplasmic reticulum (ER) stress has been proved to play a fundamental role in the pathogenesis of Alzheimer's disease, diabetes, atherosclerosis, cancer, etc. In this study, we provide the first direct evidence that ER stress may be a significant driver of morphine tolerance. Binding immunoglobulin protein (BiP), the ER stress marker, was significantly upregulated in neurons in spinal dorsal horn in rats being treated with morphine for 7 days. Additionally, chronic morphine treatment resulted in the activation of three arms of unfolded protein response (UPR): inositol-requiring enzyme 1/X-box binding protein 1 (IRE1/XBP1), protein kinase RNA-like ER kinase/eukaryotic initiation factor 2 subunit alpha (PERK/elF2 $\alpha$ ), and activating transcription factor 6 (ATF6). More importantly, inhibiting either one of the three cascades could attenuate the development of morphine tolerance. Taken together, our results suggest that ER stress in spinal cord might contribute to the development of morphine tolerance. These findings implicate a potential clinical strategy for preventing morphine tolerance and may contribute to expanding the morphine usage in clinic.

Keywords: morphine tolerance, endoplasmic reticulum stress, unfolded protein response, binding immunoglobulin protein, activating transcription factor 6, protein kinase RNA-like ER kinase, inositol-requiring enzyme 1

\section{INTRODUCTION}

Morphine is a classical agonist of $\mu$ opioid receptor, which has been widely used for the treatment of acute and chronic pain due to its potent analgesic effect. Repeated administration of morphine always lead to the drug tolerance, and patients may need higher doses of drug to achieve comparable pain relief (King et al., 2005; Williams et al., 2013). Considerable advance

\footnotetext{
Abbreviations: ATF6, activating transcription factor 6; BiP, binding immunoglobulin protein; cAMP, adenosine $3^{\prime}, 5^{\prime}$ monophosphate; CGRP, calcitonin gene-related peptide; DPN, diabetic peripheral neuropathy; eGFP, enhanced green fluorescent protein; eIF2 $\alpha$, eukaryotic initiation factor 2 subunit alpha; ER, endoplasmic reticulum; GAPDH, glyceraldehyde3-phosphate dehydrogenase; GFAP, glial fibrillary acidic protein; GRs, glucocorticoid receptors; HRP, horseradish peroxidase; Ibal, ionized calcium-binding adapter molecule 1; IRE1, inositol-requiring enzyme 1; MAPK, mitogen-activated protein kinase; MPE, maximal possible antinociceptive effect; NMDA, $N$-methyl-D-aspartate; p-eIF2 $\alpha$, phosphorylation of eIF $2 \alpha$; PERK, protein kinase RNA-like ER kinase; PFA, paraformaldehyde; PKA, protein kinase A; PKC, protein kinase C; p-PERK, phosphorylation of PERK; PVDF, polyvinylidene fluoride; SCI, spinal cord injury; SDS-PAGE, sodium dodecyl sulfatepolyacrylamide gel electrophoresis; UPR, unfolded protein response; XBP1, X-box binding protein 1; XBP1s, spliced variant of XBP1; XBP1u, unspliced XBP1.
} 
has been made in the mechanisms underlying morphine tolerance, including desensitization and internalization of opioid receptor (Narita et al., 2006; Martini and Whistler, 2007), heterodimers of $\mathrm{G}$ protein-coupled receptors (Pasternak and Pan, 2011; Szentirmay et al., 2013), involvement of chemokines (Ye et al., 2014; Guo and Gao, 2015; Guo et al., 2017; Wang et al., 2017), etc. However, detailed mechanisms are still far from clear and few strategies are available for the management of morphine tolerance for now. Therefore, further studies are needed to explore novel therapeutic targets for morphine tolerance.

Endoplasmic reticulum (ER) stress is a complex pathophysiological process, which occurs due to the disturbances of ER homeostasis such as glucose deprivation, accumulation of unfolded or misfolded proteins, and oxidative stress ( $\mathrm{Xu}$ et al., 2005; Bettigole and Glimcher, 2015). In an attempt to restore the intracellular homeostasis, ER stress could lead to the activation of UPR, which is mediated by three ER stress sensors and related signaling pathways: IRE1, PERK and ATF6 (Ron and Walter, 2007; Mori, 2015). Under physiological conditions, ER stress sensors are kept in the inactive state through being bound by chaperone BiP (Bertolotti et al., 2000). However, BiP could dissociate from the sensors when ER stress occurs, resulting in the activation of UPR. ER stress has been reported to play a fundamental role in the pathogenesis of many diseases including neurodegenerative disorders (Doyle et al., 2011), metabolic syndrome (Piperi et al., 2012), cardiovascular diseases (Liu et al., 2016) and cancer (Wang et al., 2014). And it might also be involved in the induction and maintenance of neuropathic pain (Inceoglu et al., 2015; Zhang et al., 2015) and inflammatory pain (Yang E.S. et al., 2014). Recent studies demonstrated that chronic morphine exposure could induce the oxidative stress in spinal neural cells (Cai et al., 2016; Lauro et al., 2016), and BiP mutation in mice could attenuate the development of morphine tolerance (Dobashi et al., 2010), which indicate the pivotal role of $\mathrm{BiP}$ in the mechanism of morphine tolerance. Besides, currently evidences have proved the involvement of ER stress in the pathogenesis of SCI (Penas et al., 2007; Ohri et al., 2011). However, whether ER stress is involved in the mechanism of morphine tolerance remains unknown. In this study, we sought to investigate the expressions of $\mathrm{BiP}$ and ER stress-related signaling pathways in spinal cord, and evaluate the effects of inhibiting spinal ER stress sensors during the development of morphine tolerance, to explore the potential role of ER stress in morphine tolerance.

\section{MATERIALS AND METHODS}

\section{Animals}

Adult male Sprague-Dawley rats weighing 220-250 g, were purchased from Laboratory Animal Center, Tongji Medical College, Huazhong University of Science and Technology. Animals were housed individually under controlled conditions $\left(22 \pm 0.5^{\circ} \mathrm{C}\right.$, relative humidity $40-60 \%$, standard 12: $12 \mathrm{~h}$ light: dark cycles, food and water ad libitum). All experimental procedures and protocols were reviewed and approved by Experimental Animal Care and Use Committee of Tongji Medical College, Huazhong University of Science and Technology, and carried out in accordance with the National Institutes of Health Guidelines for the Care and Use of Laboratory Animals.

\section{Intrathecal Catheterization}

For drug administration, intrathecal (i.t.) catheters were implanted using a lumbar approach, as described previously (Zhou et al., 2017). Briefly, animals were anesthetized with intraperitoneal injection (i.p.) of pentobarbital sodium (60 mg/kg). The lumbar region of rat was shaved. A sterile polyethylene catheter (PE-10; outer diameter $0.5 \mathrm{~mm}$, inner diameter $0.3 \mathrm{~mm}$; Anilab Software \& Instruments, Ningbo, China) was inserted into subarachnoid cavity between L4 and L5 vertebrae. The catheter was subcutaneously tunneled, externalized, and fixed to the back of neck. Wounds were sutured after disinfection with $75 \%$ (v/v) ethanol. Correct intrathecal placement of catheter was verified by a temporary motor block of both hind limbs after intrathecal injection of $10 \mu \mathrm{L}$ of $2 \%$ lidocaine. Rats were housed individually after surgery and allowed a 7-day recovery period before the following experiments. Rats with hind limb paralysis or paresis after surgery were excluded and euthanized with overdose of pentobarbital sodium.

\section{Drug Administration}

The drugs used in this study were prepared as follows. Morphine hydrochloride (Shenyang First Pharmaceutical Factory, China) was diluted in saline (Northeast Pharmaceutical Group, China). Specific IRE1 $\alpha$ endonuclease inhibitor STF-083010 (Selleckchem, Houston, TX, United States) and selective PERK inhibitor GSK2606414 (Selleckchem, Houston, TX, United States) were dissolved in 100\% dimethyl sulfoxide (DMSO, Sigma, St. Louis, MO, United States), respectively. STF-083010 (10 or $50 \mu \mathrm{g}$ ), GSK2606414 (10 or $100 \mu \mathrm{g}$ ) or vehicle solution was intrathecal injected $30 \mathrm{~min}$ before morphine administration in a volume of $10 \mu \mathrm{L}$, respectively, followed by $10 \mu \mathrm{L}$ of saline to flush the catheter. The doses of STF-083010 and GSK2606414 used in this study were determined according to our preliminary results.

\section{Morphine Tolerance}

Morphine tolerance was induced by intrathecal administration of morphine (10 $\mu \mathrm{g}$, twice daily) for 7 days, as described previously (Guo et al., 2016). Rats in the control group received an equivalent volume of saline at the same time points. The development of morphine tolerance was assessed by behavioral tests on days 1, 3, 5, and 7 (Peng et al., 2017).

\section{Behavioral Assessment}

Thermal pain thresholds in rats were measured by a tail-flick latency test before drug administration and at $30 \mathrm{~min}$ after morphine administration on days 1, 3, 5, and 7 (Cui et al., 2008). Briefly, rat was placed in a plastic container. The body of rat was restrained and one-third to the tip of tail was immersed into water which was maintained at $50 \pm 0.2^{\circ} \mathrm{C}$. A positive response was defined as the rapid removal of tail from hot water. A cutoff time of $15 \mathrm{~s}$ was determined to prevent tail damage. The test was repeated three times with an interval of $5 \mathrm{~min}$ and 
the mean of three tests was considered as the final latency. The percentage of maximal possible antinociceptive effect (\%MPE) was calculated by comparing the test latency before (baseline, $\mathrm{BL}$ ) and after drug injection (TL) using the following equation: $\% \mathrm{MPE}=[(\mathrm{TL}-\mathrm{BL}) /($ cutoff time $-\mathrm{BL})] \times 100$. All the behavioral tests were performed by an investigator who was blinded to the experimental design.

\section{Quantitative Real-Time Polymerase Chain Reaction (qRT-PCR)}

Rats were deeply anesthetized with pentobarbital sodium (60 mg/kg, i.p.) and L3-L5 spinal cord segments were quickly removed. Total RNA of spinal cord tissue from each group was extracted using Trizol Reagent (Invitrogen, Carlsbad, CA, United States) according to the manufacturer's instructions and then synthesized to cDNA by reverse transcription. StepOne Real-Time PCR System (Applied Biosystems, Carlsbad, CA, United States) was used to conduct the qRT-PCR. One microgram of total RNA from each sample was added into $20 \mu \mathrm{L}$ reactive solution of reverse transcription, respectively. The specific primer sequences for target genes in this study were designed and synthesized by Takara Biomedical Technology (Kyoto, Japan) and listed in Table 1. Housekeeping gene GAPDH was used as an internal control. Relative quantification of mRNA was performed by $2^{-\Delta \Delta C \mathrm{t}}$ method.

\section{Western Blots}

Rats were deeply anesthetized with sodium pentobarbital (60 mg/kg, i.p.) and L3-L5 spinal cord segments were quickly removed. Total proteins of spinal cord tissue from each group were extracted using RIPA lysis buffer combined with a mixture of proteinase inhibitors according to the manufacturer's instruction (Boster, Wuhan, China). The protein concentration of supernatants was measured by using bicinchoninic acid assay. $50 \mu \mathrm{g}$ protein from each sample was loaded and separated on $10 \%$ SDS-PAGE. Electrophoresis was conducted at $60 \mathrm{~V}$ constant voltage for stacking gel and $100 \mathrm{~V}$ for separating gel. The proteins were subsequently electro-transferred (200 mA, 60$90 \mathrm{~min}$ ) to a PVDF membrane (IPVH00010, Millipore, Bellerica, MA, United States). The membranes were blocked with 5\% bovine serum albumin for $1 \mathrm{~h}$ at room temperature (RT) and then incubated overnight at $4^{\circ} \mathrm{C}$ with the following primary antibodies: rabbit anti-BiP antibody (1:4000; ab21685; Abcam, Cambridge, MA, United States), rabbit anti-IRE1 antibody (1:4000; ab37073; Abcam, Cambridge, MA, United States), rabbit anti-XBP1 antibody (1:2000; A1731; ABclonal, Wuhan, China), rabbit anti-PERK (C33E10) antibody (1:1000; \#3192; CST, Beverly, MA, United States), rabbit anti-phospho-PERK (Thr980) (16F8) antibody (1:1000; \#3179; CST, Beverly, MA, United States), rabbit anti-eIF2 $\alpha$ (D7D3) antibody (1:2000; \#5324; CST, Beverly, MA, United States), rabbit anti-phosphoeIF2 $\alpha$ (Ser51)(D9G8) antibody (1:1000; \#3398; CST, Beverly, MA, United States), rabbit anti-ATF6 antibody (1:1000; ab203119; Abcam, Cambridge, MA, United States) and rabbit anti-GAPDH antibody (1:5000; AS1039; Aspen, Wuhan, China). After being thoroughly washed, the membranes were incubated with HRP - conjugated goat anti-rabbit IgG (1:5000, Aspen, Wuhan, China) for $2 \mathrm{~h}$ at RT. Finally, proteins were detected by SuperLumia ECL Plus HRP Substrate Kit (K22030, Abbkine, Wuhan, China) and a computerized image analysis system (Bio-Rad, ChemiDoc XRS+, United States). Image Lab software (Bio-Rad Laboratories) was used to quantify the intensity of protein blots, which were normalized to loading control GAPDH and expressed as the fold of control. The blot density of control group was set as 1 .

\section{Immunofluorescent Staining}

After being treated with morphine or saline for 7 days, rats were deeply anesthetized with sodium pentobarbital $(60 \mathrm{mg} / \mathrm{kg}$, i.p.) and perfused intracardially with saline followed by $4 \%$ ice-cold PFA in 0.1 M phosphate buffer saline (PBS). After perfusion, L3L5 spinal cord segments were quickly removed and post-fixed in $4 \%$ PFA for $4 \mathrm{~h}$ and subsequently dehydrated in $30 \%$ sucrose solution for 2 days at $4^{\circ} \mathrm{C}$. Transverse spinal sections $(20 \mu \mathrm{m})$ were cut in a cryostat (CM1900, Leica, Germany), mounted on poly-lysine-coated slides, and stored at $-80^{\circ} \mathrm{C}$ until use.

\section{Single Immunostainings}

The sections were penetrated with $0.3 \%$ Triton-X-100 for $15 \mathrm{~min}$ and blocked with $10 \%$ donkey serum for $1 \mathrm{~h}$ at RT. The sections were then incubated overnight at $4^{\circ} \mathrm{C}$ with the following primary antibodies: rabbit anti-BiP antibody (1:500; ab21685; Abcam, Cambridge, MA, United States), rabbit anti-IRE1 antibody (1:50; ab37073; Abcam, Cambridge, MA, United States), rabbit anti-XBP1 antibody (1:50; A1731; ABclonal, Wuhan, China), rabbit anti-PERK (phospho T981) antibody (1:50; YP1055; ImmunoWay, Plano, TX, United States), rabbit anti-phospho-eIF2 $\alpha$ (Ser51) (D9G8) antibody (1:50; \#3398; CST, Beverly, MA, United States), rabbit anti-ATF6 antibody (1:50; ab203119; Abcam, Cambridge, MA, United States). Then, the sections were incubated with Alexa Fluor 488labeled donkey anti-rabbit secondary antibody (1:300; A21206; Invitrogen, Carlsbad, CA, United States) for $2 \mathrm{~h}$ at RT and washed with PBS. Sections were rinsed, mounted, and cover-slipped with 50\% glycerol. Images were captured using a fluorescence microscope (DM2500, Leica, Germany). $\mathrm{BiP} / \mathrm{IRE} 1 / \mathrm{XBP} 1 / \mathrm{p}-\mathrm{PERK} / \mathrm{p}$-eIF2 $\alpha / \mathrm{ATF} 6$-immunolabeled surface areas were measured in laminae I-IV of spinal cord dorsal horn using Image Pro Plus 4 software (Media Cybernetics, Maryland, MD, United States). Quantification of immunoreactivity was accomplished by calculating the percentages of immunostaining [(positive immunofluorescent surface area)/(total measured picture area $\times 100$ ]. Three rats of each group were used for statistical analysis. All the image analyses were performed by an investigator who was blinded to the experimental design.

\section{Double Immunofluorescent Staining}

For double immunofluorescence, the sections were incubated with a mixture of two primary antibodies followed by a mixture of Alexa 488-conjugated and Alexa 594-conjugated secondary antibodies (1:300; A-21207; Invitrogen, Carlsbad, CA, United States). Specifically, to identify the cell types expressing BiP, IRE1, XBP1, PERK (phospho T981), Phospho-eIF2 $\alpha$ (Ser51) or ATF6, each of the primary antibodies was mixed with the 
TABLE 1 | Primers for Real-time PCR.

\begin{tabular}{|c|c|c|c|}
\hline Name & Primer & Sequence & Size \\
\hline \multirow[t]{2}{*}{ Rat GAPDH } & Forward & 5' - ACAGCAACAGGGTGGTGGAC -3' & 253 bp \\
\hline & Reverse & $5^{\prime}$ - TाTGAGGGTGCAGCGAACTT -3' & \\
\hline \multirow[t]{2}{*}{ Rat ATF6 } & Forward & 5' - AGCCCCTCATTAACACGACA -3' & $158 \mathrm{bp}$ \\
\hline & Reverse & 5' - AGAATTCGAGCCCTGTTCCA -3’ & \\
\hline \multirow[t]{2}{*}{ Rat IRE1 } & Forward & 5' - CGGGAGAGCTGTGGTTAAGA -3’' & 244 bp \\
\hline & Reverse & 5' - TCGGTAGGTGTGAGAGAGGA -3' & \\
\hline \multirow[t]{2}{*}{ Rat XBP1u } & Forward & 5' - AGACTACGTGCGCCTCTGCA -3' & 170 bp \\
\hline & Reverse & 5' - AGACTCTGGGGAAGGACATT -3' & \\
\hline \multirow[t]{2}{*}{ Rat XBP1s } & Forward & 5' - GCTITCATCCAGCCATTGTCT -3' & $150 \mathrm{bp}$ \\
\hline & Reverse & 5' - AGTTCGTTGGCAAAAGTGTC -3' & \\
\hline \multirow[t]{2}{*}{ Rat PERK } & Forward & 5' - AGTCGGTCTITCTCAGTGGG -3' & $160 \mathrm{bp}$ \\
\hline & Reverse & 5' - CCATGTCGCAATCTGTCAGG -3' & \\
\hline \multirow[t]{2}{*}{ Rat elF2 $\alpha$} & Forward & 5' - CTCGCAACGCAGCATTCTAT -3' & $187 \mathrm{bp}$ \\
\hline & Reverse & 5' - GCACACGTGGCTGTTAAGAT -3' & \\
\hline \multirow[t]{2}{*}{ Rat BiP } & Forward & 5' - GAACCAACTCACGTCCAACC -3' & $247 \mathrm{bp}$ \\
\hline & Reverse & 5'-CTाTCCCAAATACGCCTCGG -3' & \\
\hline
\end{tabular}

antibody of neuronal marker neuronal nuclei (NeuN; 1:50; MAB377; Millipore, Billerica, MA, United States), astrocytic marker GFAP (1:200; \#3670; CST, Beverly, MA, United States), or microglial marker Iba1 (1:100; ab5076; Abcam, Cambridge, MA, United States), respectively. Images were captured with a fluorescence microscope (DM2500, Leica, Germany). Nonspecific staining was determined by omitting the primary antibodies.

\section{Preparation of ATF6 RNAi-Lentivirus}

According to the previous literatures (Seo et al., 2008, 2010), recombinant shRNA lentiviral vectors targeting rat ATF6 gene (ATF6 RNAi-LV) and non-specific control lentivirus (NC-LV) were designed by Genechem (Shanghai, China). Briefly, ATF6 $\left(5^{\prime}\right.$-CCATTGTGTTACCAGCAAT- $\left.3^{\prime}\right)$ or non-specific control shRNA oligonucleotides (5'-TTCTCCGAACGTGTCACGT-3') were inserted into lentivirus transfer vector (GV248-LV, GeneChem Co. Ltd., Shanghai, China) under the control of U6 promoter. Lentiviruses were acquired from triple-infected 293T cells with approximately $80 \%$ confluence. The lentiviral vector backbone was hU6-MCS-Ubiquitin-EGFP-IRES-puromycin. The pellets were suspended in DMEM, aliquoted, and stored at $-80^{\circ} \mathrm{C}$. The viral titer for stock was $3.0 \times 10^{8} \mathrm{TU} / \mathrm{mL}$ medium.

\section{Stereotaxic Injection of RNAi-Lentivirus Into Spinal Dorsal Horn}

Stereotaxic injection of lentiviral vectors into spinal dorsal horn was conducted 3 days before intrathecal catheterization according to the methods described previously (Fu et al., 2016). Briefly, rats were anesthetized with pentobarbital sodium (60 mg/kg, i.p.). The lumbar region of rat was shaved. A longitudinal skin incision was performed along the midline of lumbar spine to cautiously expose the L1 vertebrae. Two holes of $1 \mathrm{~mm}$ diameter were carefully drilled on the left and right of vertebrae $(0.5 \mathrm{~mm}$ from the midline). Then, rats were placed on a stereotaxic instrument. The tip of a Hamilton microsyringe was insert into spinal dorsal horn of rats through each hole on the vertebrae at a depth of $0.8 \mathrm{~mm}$. $2 \mu \mathrm{L}$ of ATF6 RNAi-LV or NC-LV solution was administered through each hole at a rate of $0.5 \mu \mathrm{L} / \mathrm{min}$, respectively. The needle was left in spinal cord for at least 2 min before removed. After surgery, rats were kept in an insulation can for $30 \mathrm{~min}$ for complete recovery from anesthesia.

\section{Statistical Analyses}

Animal sample size for behavioral experiment was decided by power analysis using SSize2021 software (National University of Singapore, Singapore) (version 2). With anticipated population proportion $\mathrm{P} 1=0.95, \mathrm{P} 2=0.05$, significance level 0.05 and power of test 0.09 , the sample size was estimated to be four per group. All data were presented as mean \pm SEM and analyzed using GraphPad Prism 5 (GraphPad Software Inc.). Behavioral test was analyzed by two-way repeated measure ANOVA (treatment group $\times$ time) to detect overall differences among treatment groups followed by Bonferroni's test to detect the changes of $\% \mathrm{MPE}$ after drug injection over time. The results of qRTPCR and western blots were analyzed by one-way ANOVA followed by Bonferroni post hoc test. Individual comparisons were conducted with unpaired $t$-test. $p<0.05$ was considered statistically significant.

\section{RESULTS}

\section{Chronic Morphine Treatment Induced Drug Tolerance and Increased BiP Expression in Spinal Cord}

Rats were intrathecal administered with morphine $(10 \mu \mathrm{g} / 5 \mu \mathrm{L})$ or saline $(5 \mu \mathrm{L})$ twice daily for consecutive 7 days. Behavioral tests were conducted before drug administration and at $30 \mathrm{~min}$ after the last drug administration on days 1, 3, 5, and 7 . As shown in Figure 1A. This resulted in reliable interaction between morphine and time $\left(F_{3,45}=12.81\right)$, morphine and 
A

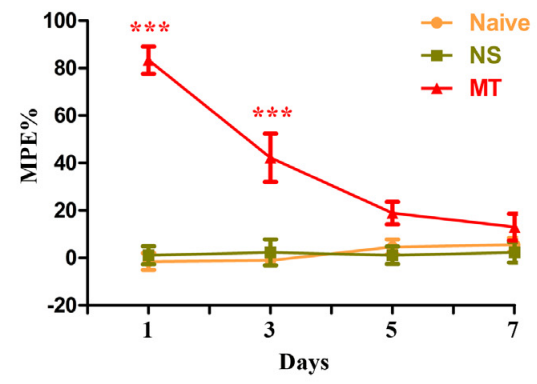

C

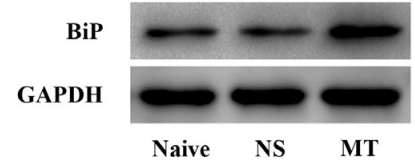

E
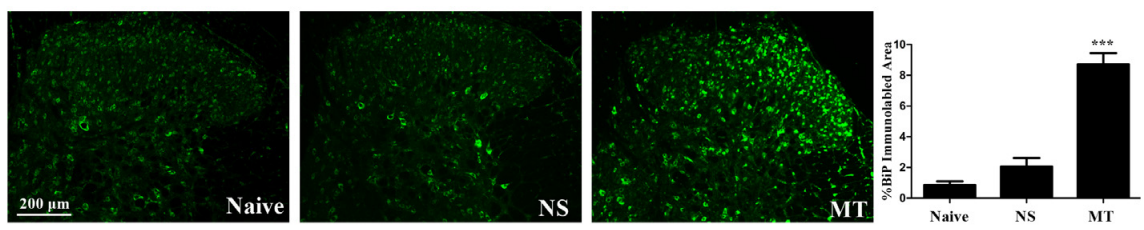

$\mathbf{F}$
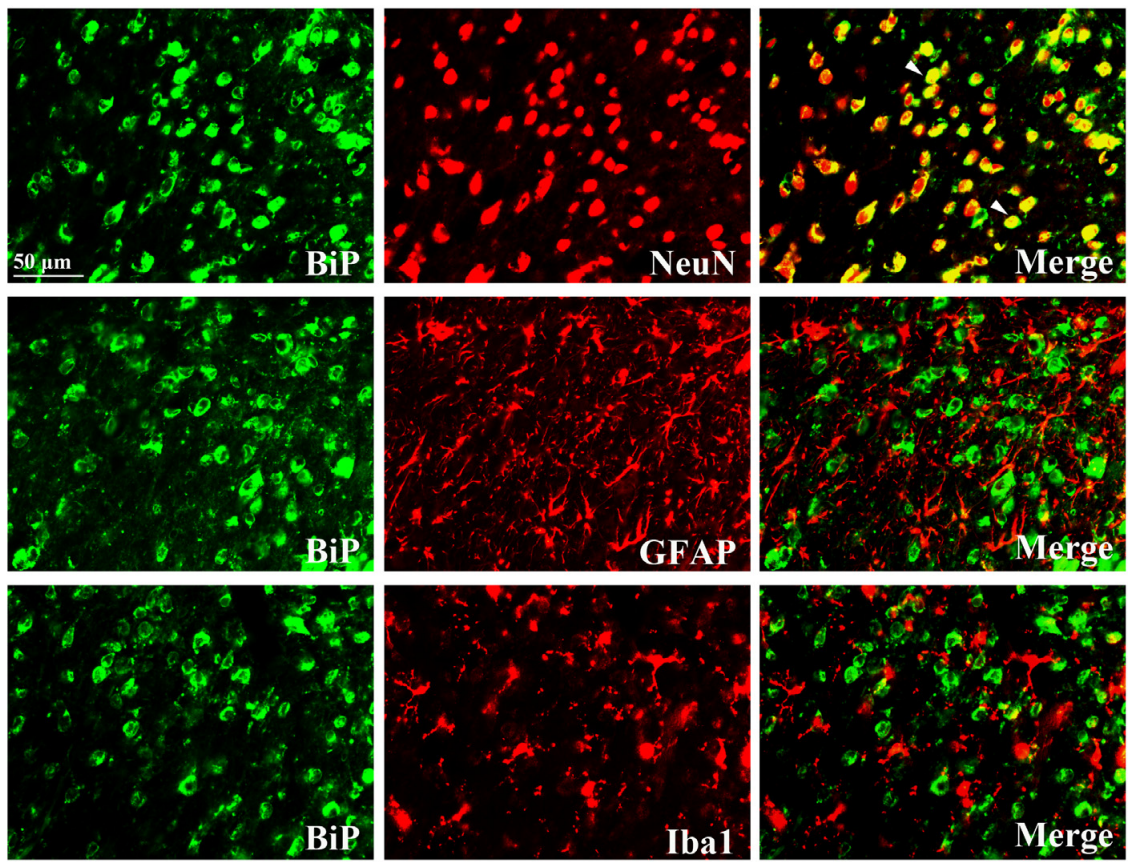
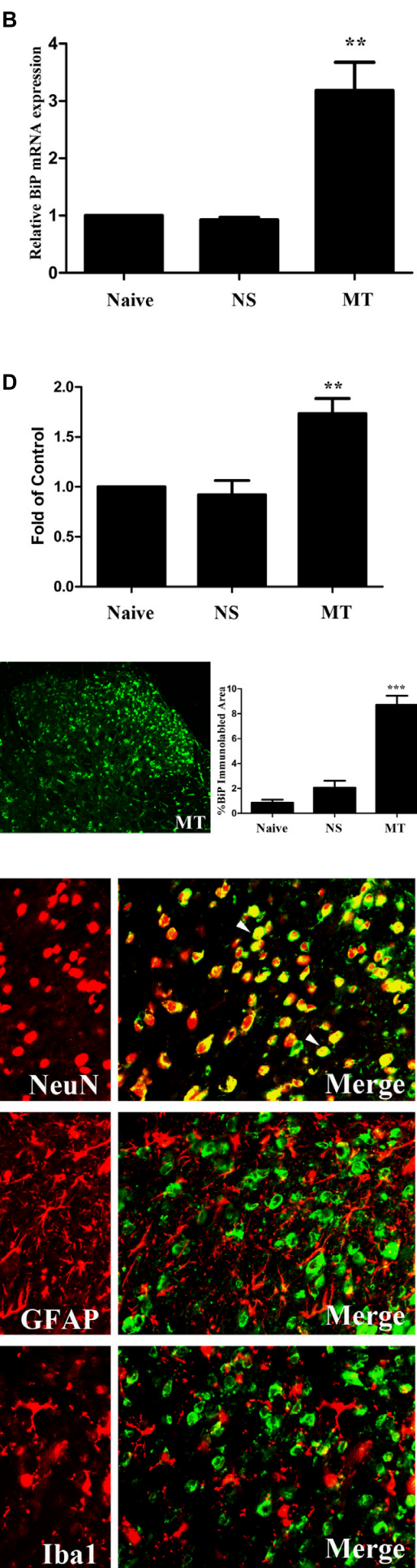

FIGURE 1 | Expression of BiP in lumbar spinal cord. (A) Thermal pain threshold of rats was assessed using the percentage of maximal possible antinociceptive effect (\%MPE) according to the tail-flick latency of rats. The \%MPE in rats received morphine (10 $\mu \mathrm{g}$, twice daily, intrathecally) on days 5 and 7 were dramatically decreased compared with the baseline on day 1 . ( ${ }^{* * *} p<0.001$ compared with naive rats, $n=6$ in each group). (B-D) The expression of BiP was significantly increased in morphine-tolerant rats measured by real-time PCR and western blots, respectively. ( ${ }^{* *} p<0.01$ compared with naive rats, $n=4$ in each group). (E) Immunostaining of BiP in spinal dorsal horn. BiP expression was significantly increased in morphine-tolerant rats compared with those in naiive rats. $\left({ }^{* * *} p<0.001\right.$ compared with naive rats, $n=3$ in each group, scale bar: $\left.200 \mu \mathrm{m}\right)$. (F) Double immunostaining of BiP and cell-specific markers in morphine-tolerant rats. BiP was co-localized with NeuN (indicated by arrows). Scale bar: $50 \mu \mathrm{m}$. BiP, binding immunoglobulin protein; NS, normal saline; MT, morphine tolerance. 
saline $\left(F_{2,45}=49.91\right)$, \% MPE in rats received morphine were significantly higher than those in saline-treated rats on days 1 and $3(p<0.001)$. There was no statistically significant difference in $\% \mathrm{MPE}$ levels between morphine-treated and saline-treated rats on days 5 and 7 ( $p>0.05)$, indicating that chronic morphine tolerance had been successfully established.

To investigate the involvement of ER stress in morphine tolerance, we first examined the spinal expression of $\mathrm{BiP}$, a marker of ER stress. On day 7, increased expressions of $\operatorname{BiP}$ mRNA $\left(F_{2,11}=20.01\right)$ and protein $\left(F_{2,11}=14.21\right)$ were detected in spinal cord of rats treated with morphine (Figures 1B-D, $p<0.01$ compared to naïe and saline-treated rats), indicating that ER stress might be induced by chronic morphine treatment. Next, we examined the distribution and cellular localization of $\mathrm{BiP}$ in spinal cord. The results showed that BiP was extensively expressed in spinal dorsal horn (Figure 1E). The immunoreactivity of $\mathrm{BiP}$ in morphine-tolerant rats was significantly higher than those in naïve and saline-treated rats
$\left(F_{2,8}=61.49, p<0.001\right)$. And $\mathrm{BiP}$ was found to be colocalized with neuronal marker NeuN, not astrocytic marker GFAP or microglial marker Ibal (Figure 1F). These indicate that upregulation of $\mathrm{BiP}$ expression in neurons in spinal dorsal horn might be involved in the development of morphine tolerance.

\section{Spinal IRE1/XBP1 Cascade Contributed to the Development of Morphine Tolerance}

Three ER stress sensors (IRE1, PERK, and ATF6) implement the UPR pathways. We first investigated the expressions of IRE1/XBP1 cascade during the development of morphine tolerance. The expressions of IRE1, XBP1s, XBP1u were examined on day 7 of morphine administration. As shown in Figure 2, both mRNA and protein levels of IRE1, XBP1s, and XBP1u were significantly increased in morphine-treated rats compared with naïve and saline-treated rats $\left(F_{2,11}=9.742\right.$ for

\section{A}

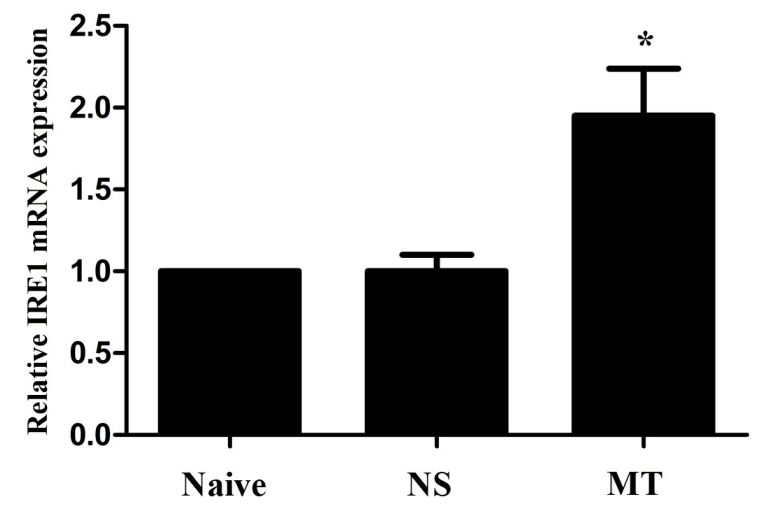

C

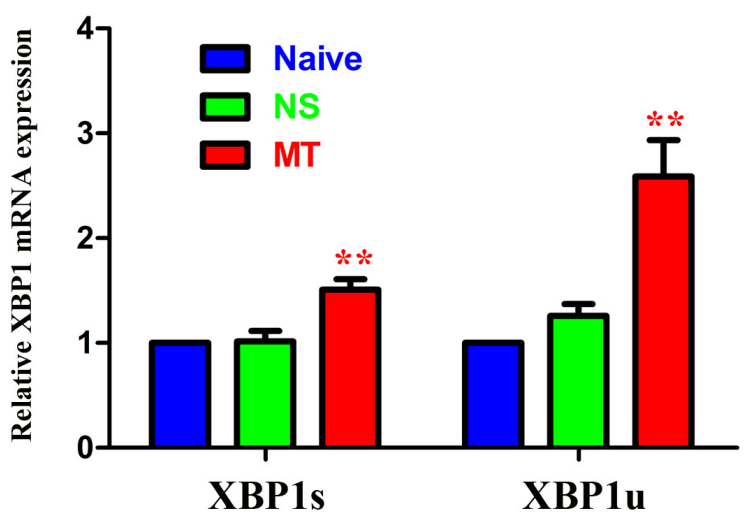

B

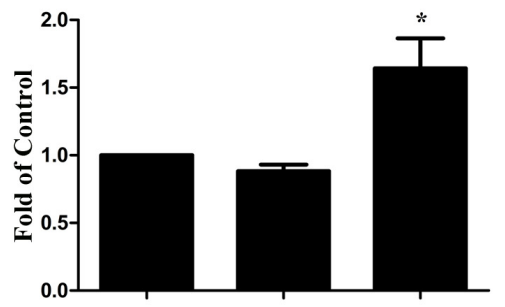

IRE1

GAPDH

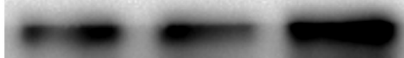

$110 \mathrm{kDa}$

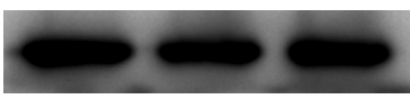

37 kDa

Naive NS MT

D
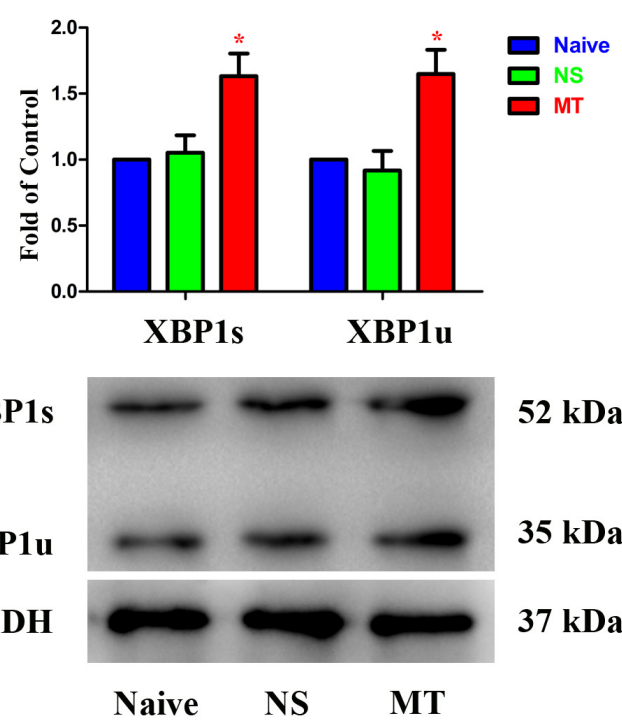

XBP1s

52 kDa

35 kDa

XBP1u

37 kDa

FIGURE 2 | Expressions of IRE1/XBP1 cascade in spinal cord. The expressions of IRE1 (A,B), XBP1s and XBP1u (C,D) were significantly increased in morphine-tolerant rats measured by real-time PCR and western blots, respectively. $\left({ }^{*} p<0.05,{ }^{* *} p<0.01\right.$ compared with naive rats, $n=4$ in each group) IRE1: inositol-requiring enzyme 1. XBP1, X-box binding protein 1; XBP1s, spliced variant of XBP1; XBP1u, unspliced XBP1; NS, normal saline; MT, morphine tolerance. 
A

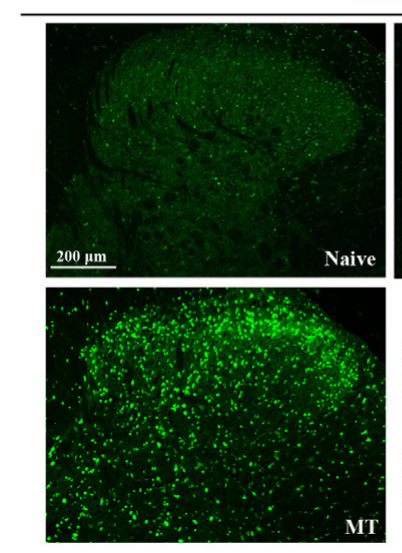

RE1

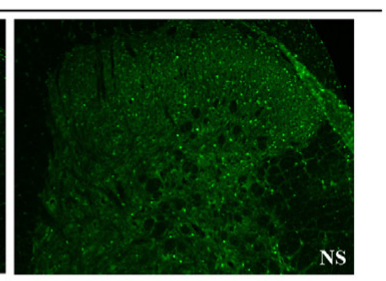

MT

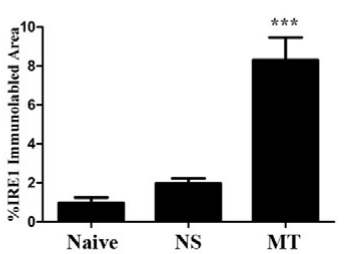

B
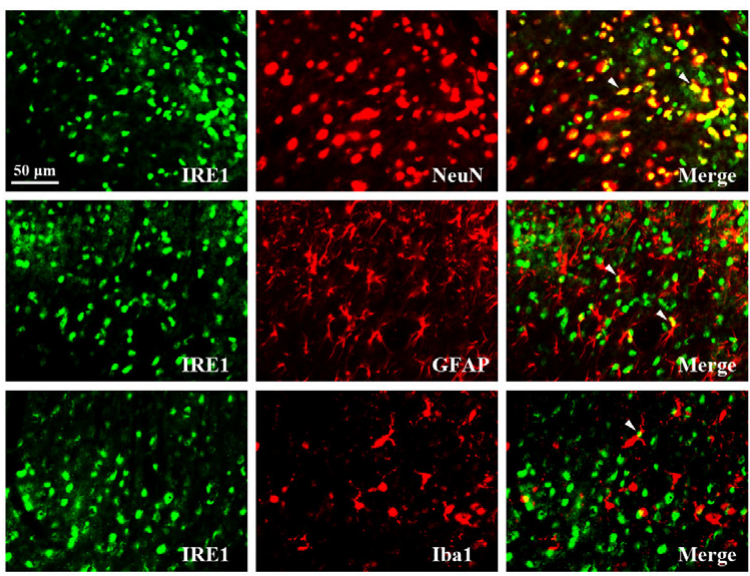

C

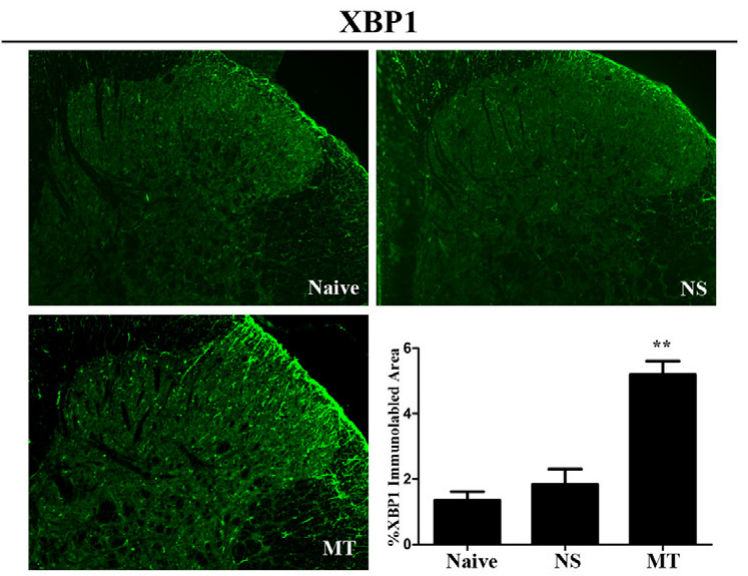

D
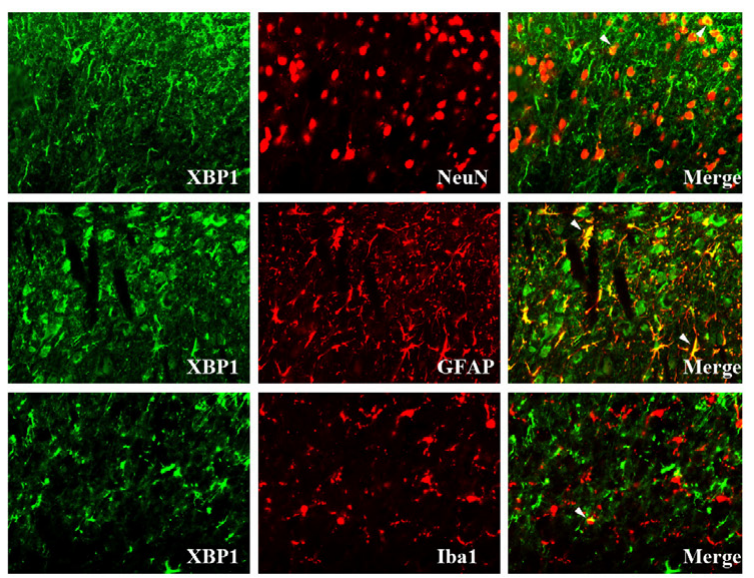

FIGURE 3 | Distributions and cellular localizations of IRE1 and XBP1 in spinal cord. (A,C) Immunostaining of IRE1 and XBP1 in spinal dorsal horn. IRE1 and XBP1 were extensively expressed in spinal dorsal horn. The immunoreactivity of IRE1 and XBP1 were significantly increased in morphine-tolerant rats. ${ }^{* *} p<0.01$, ${ }^{* * *} p<0.001$ compared with naive rats, $n=3$ in each group, scale bar: $\left.200 \mu \mathrm{m}\right)$. (B,D) Double immunostaining of IRE1 or XBP1 and cell-specific markers in morphine-tolerant rats. IRE1 and XBP1 were both mainly co-localized with NeuN and GFAP, and a minority with Iba1 (indicated by arrows). Scale bar: $50 \mu \mathrm{m}$. IRE1, inositol-requiring enzyme 1; XBP1, X-box binding protein 1; NS, normal saline; MT, morphine tolerance.

mRNA of IRE1, $F_{2,11}=13.14$ for mRNA of XBP1s, $F_{2,11}=16.56$ for mRNA of XBP1u, $F_{2,11}=9.709$ for protein of IRE1, $F_{2,11}=7.884$ for protein of XBP1s, $F_{2,11}=8.723$ for protein of XBP1u, $p<0.05)$, as well as the immunoreactivity of IRE1 (Figure 3A, $F_{2,8}=31.84, p<0.001$ ) and XBP1 (Figure 3C, $\left.F_{2,8}=28.07, p<0.01\right)$ in spinal dorsal horn. To determine the cellular localization of IRE1 and XBP1 in spinal cord, double immunofluorescent staining was performed in morphine-treated rats. The results showed that IRE1 and XBP1 were mainly colocalized with NeuN and GFAP, and a minority with Iba1 in spinal dorsal horn (Figures 3B,D). These results indicate that chronic morphine treatment could result in the activation of IRE1/XBP1 cascade mainly in neurons and astrocytes in spinal cord.

To further investigate the role of spinal IRE1/XBP1 cascade in the development of morphine tolerance, a specific IRE1 $\alpha$ endonuclease inhibitor STF-083010 was intrathecal injected $30 \mathrm{~min}$ before morphine administration. As shown in Figure 4A, a single dose of STF-083010 (10 $\mu \mathrm{g}$ or $50 \mu \mathrm{g})$ did not affect the antinociceptive effect of morphine on day 1 of morphine administration $(p>0.05)$, but consecutive treatments with STF$083010(50 \mu \mathrm{g}$, but not $10 \mu \mathrm{g}$ ) could significantly attenuate the development of morphine tolerance from days 3 to 7 $\left(F_{1,24}=0.046\right.$, for $10 \mu \mathrm{g}$ STF-083010, $p>0.05 ; F_{1,24}=14.03$, for $50 \mu \mathrm{g}$ STF-083010, $p<0.05)$. Moreover, the increased expressions of XBP1s and XBP1u induced by chronic morphine treatment could be inhibited by STF-083010 pretreatment (Figure 4B, $F_{3,15}=22.35$, for protein of XBP1s, $F_{3,15}=8.566$ for protein of XBP1u, $p<0.05)$. Taken together, these results suggest that activation of spinal IRE1/XBP1 cascade in neurons and astrocytes might be involved in the development of morphine tolerance.

\section{Spinal PERK/elF2 $\alpha$ Cascade Contributed to the Development of Morphine Tolerance}

To investigate the expressions of PERK/eIF2 $\alpha$ cascade during the development of morphine tolerance, rats were treated with 

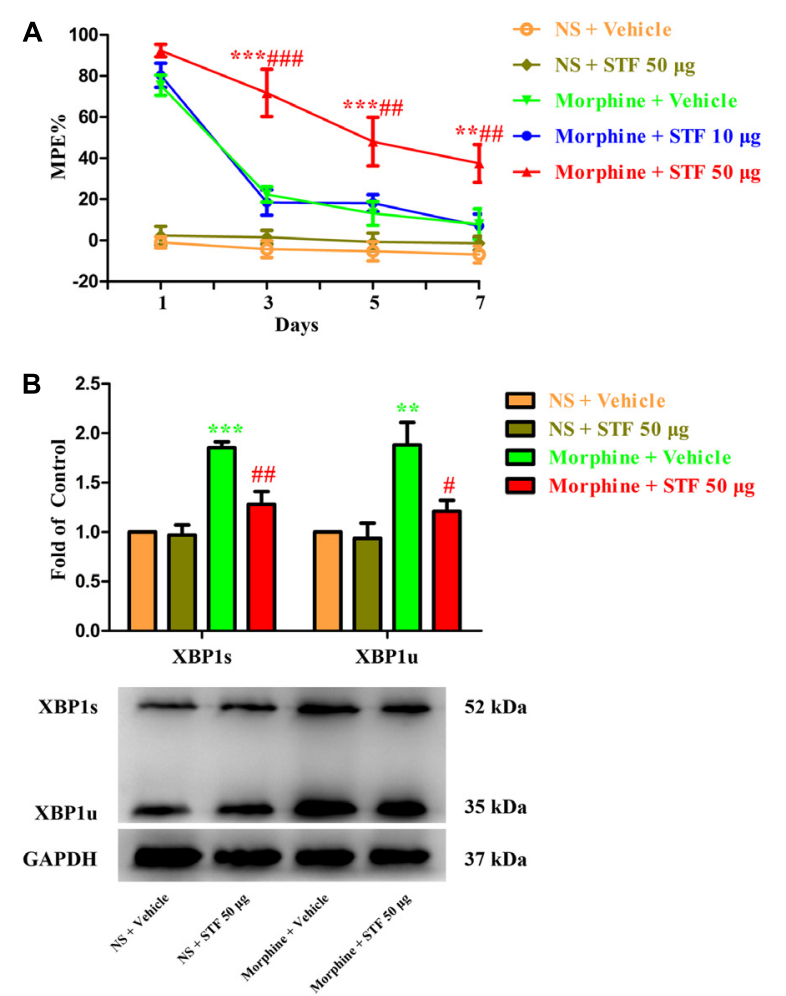

FIGURE 4 | Effect of IRE1 inhibitor on the development of morphine tolerance. Specific IRE1 $\alpha$ endonuclease inhibitor STF-083010 (10 $\mu \mathrm{g}$ or $50 \mu \mathrm{g}$ ) was intrathecal injected 30 min before morphine treatment for 7 days, respectively. (A) Pretreatment with $50 \mu \mathrm{g}$ STF-083010 could attenuate the development of morphine tolerance. $\left({ }^{* *} p<0.01\right.$, ${ }^{* * *} p<0.001$ compared

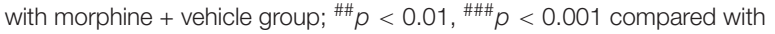
morphine + STF $10 \mu \mathrm{g}$ group, $n=5$ in each group). (B) Pretreatment with 50 $\mu \mathrm{g}$ STF-083010 could inhibit the up-regulation of XBP1s and XBP1u induced by chronic morphine treatment measured by western blots. ${ }^{* *} p<0.01$, ${ }^{* * *} p<0.001$ compared with NS + vehicle group; ${ }^{\#} p<0.05,{ }^{\# \#} p<0.01$ compared with morphine + vehicle group, $n=4$ in each group). \%MPE, percentage of maximal possible antinociceptive effect; NS, normal saline; STF, STF-083010; XBP1, X-box binding protein 1; XBP1s, spliced variant of XBP1; XBP1u, unspliced XBP1.

morphine or saline for 7 days. And the mRNA levels of PERK (Figure 5A, $F_{2,11}=26.03, p<0.001$ ) and eIF2 $\alpha$ (Figure 5C, $\left.F_{2,11}=18.64, p<0.01\right)$ were detected to be increased in morphine-treated rats. Consistently, the protein levels of PERK $\left(F_{2,11}=8.539\right)$ and $\operatorname{eIF} 2 \alpha\left(F_{2,11}=8.66\right)$ were also increased in morphine-treated rats (Figures 5B,D, $p<0.05$ ), as well as the p-PERK (Figure 5B, $F_{2,11}=5.875, p<0.05$ ) and eIF2 $\alpha$ $(\mathrm{p}-\mathrm{eIF} 2 \alpha)$ (Figure 5D, $\left.F_{2,11}=11.21, p<0.05\right)$. The results of immunofluorescent staining also confirmed the increased immunoreactivity of p-PERK (Figure 6A, $F_{2,8}=14.43, p<0.05$ ) and p-eIF2 $\alpha$ (Figure 6C, $F_{2,8}=13.05, p<0.05$ ) in spinal dorsal horn induced by chronic morphine treatment. To determine the cellular localizations of p-PERK and p-eIF $2 \alpha$ in spinal cord, double immunofluorescent staining was performed in morphinetreated rats. The results showed that p-PERK was co-localized with GFAP (Figure 6B), while p-eIF2 $\alpha$ was mainly co-localized with NeuN and Iba1, and a minority with GFAP (Figure 6D). These results indicate that chronic morphine treatment could result in the activation of PERK/eIF $2 \alpha$ cascade which may play a fundamental role in neuron-glia and astrocyte-microglia interactions in the mechanism of morphine tolerance.

To further investigate the role of spinal PERK/eIF2 $\alpha$ cascade in the development of morphine tolerance, a specific PERK inhibitor GSK2606414 was intrathecal injected $30 \mathrm{~min}$ before morphine administration. As shown in Figure 7A, a single dose of GSK2606414 (10 $\mu \mathrm{g}$ or $100 \mu \mathrm{g})$ did not affect the antinociceptive effect of morphine on day 1 of morphine administration $(p>0.05)$, but consecutive treatments with GSK2606414 (100 $\mu \mathrm{g}$, but not $10 \mu \mathrm{g})$ could significantly attenuate the development of morphine tolerance from days 3 to 7 $\left(F_{1,30}=1.639\right.$, for $10 \mu \mathrm{g}$ GSK2606414, $p>0.05 ; F_{1,30}=11.24$, for $100 \mu \mathrm{g}$ GSK2606414, $p<0.05)$. Moreover, the increased expressions of PERK, p-PERK, eIF2 $\alpha$, and p-eIF2 $\alpha$ induced by chronic morphine treatment could be inhibited by GSK2606414 pretreatment (Figures $7 \mathbf{B}, \mathbf{C}, F_{3,23}=7.225$, for protein of PERK, $F_{3,23}=10.04$ for protein of p-PERK, $p<0.05 ; F_{3,23}=8.26$, for protein of eIF $2 \alpha, F_{3,23}=11.84$ for protein of p- eIF2 $\alpha, p<0.05$ ). Taken together, these results suggest that PERK/eIF $2 \alpha$ cascade might be involved in the development of morphine tolerance via mediating interactions between neural cells in spinal cord.

\section{Spinal ATF6 Contributed to the Development of Morphine Tolerance}

To investigate the expression of ATF6 during the development of morphine tolerance, rats were treated with morphine or saline for 7 days. And both the mRNA (Figure 8A, $F_{2,11}=13.57$, $p<0.01$ ) and protein (Figure 8B, $F_{2,17}=15.83, p<0.001$ ) levels of ATF6 was increased in morphine-treated rats. The results of immunofluorescent staining also confirmed the increased immunoreactivity of ATF6 (Figure 8C, $F_{2,8}=18.54, p<0.01$ ) in spinal dorsal horn induced by chronic morphine treatment. To determine the cellular localization of ATF6 in spinal cord, double immunofluorescent staining was performed in morphine-treated rats. The results showed that ATF6 was co-localized with NeuN, not with GFAP or Iba1 (Figure 8D). As a transcription factor, ATF6 was found to be translocated from cytoplasm to nucleus after chronic morphine treatment, while it was only expressed in cytoplasm in naive rats (Figure $\mathbf{8 E}$ ). These results indicate that chronic morphine treatment result in the activation of ATF6 in neurons in spinal cord and promote the translocation of ATF6 to nucleus where it could induce the transcription of certain molecules.

To further investigate the role of ATF6 in the development of morphine tolerance, a recombinant shRNA lentiviral vector was used to knockdown the expression of ATF6 in spinal cord. $4 \mu \mathrm{L}$ of RNAi-LV or NC-LV was injected into lumbar spinal cord of rats 3 days before intrathecal catheterization, respectively. The successful lentivirus transfection in spinal dorsal horn was verified by the expression of eGFP in RNAiLV and NC-LV 1 week after microinjection (Figure 9A). The increased expressions of ATF6 in spinal cord, which were induced by chronic morphine treatment, could be inhibited by the 


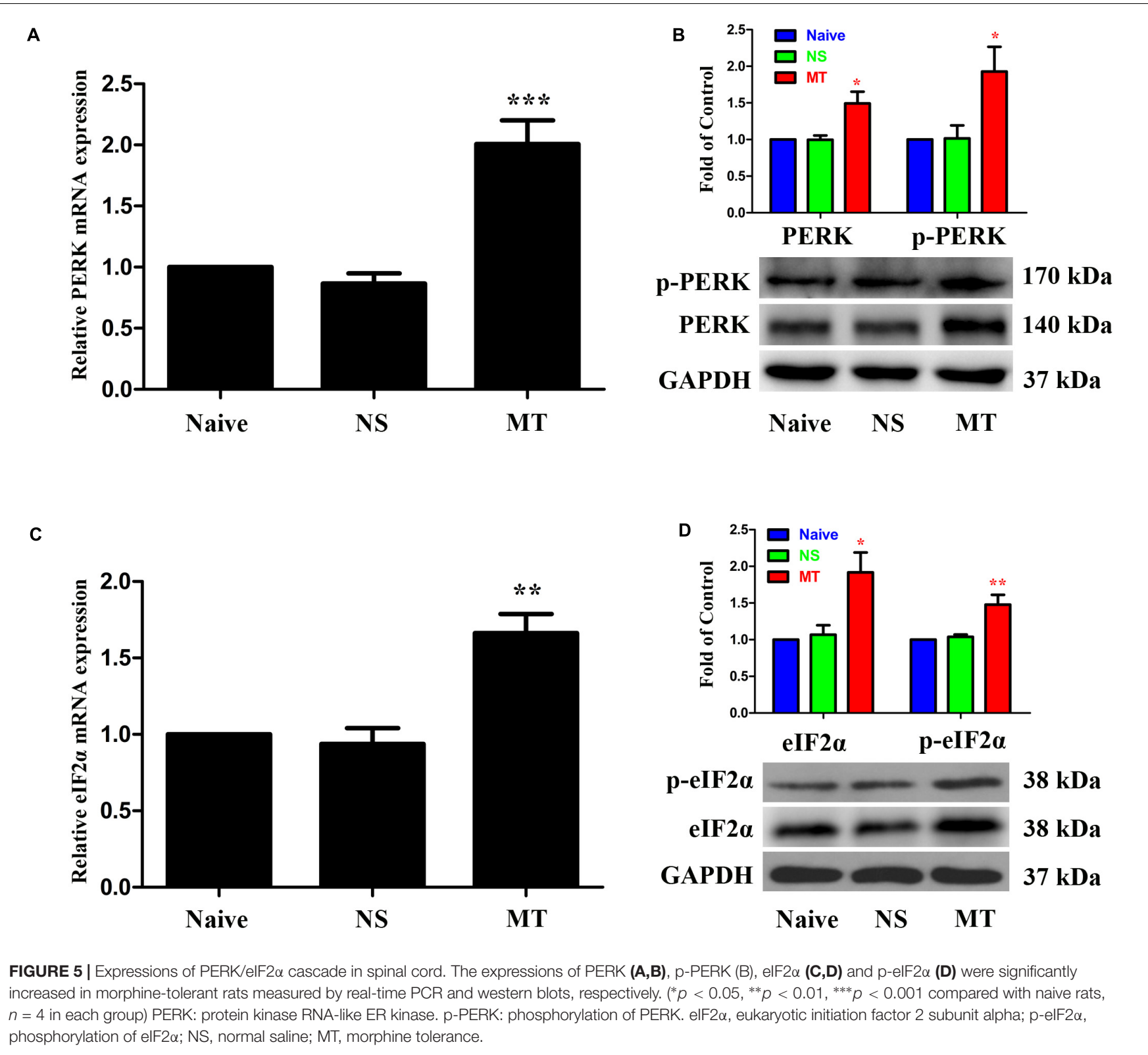

transfection of RNAi-LV (Figures 9B,C, $F_{3,15}=11.59$ for mRNA of ATF6, $F_{3,23}=10.69$ for protein of ATF6, $\left.p<0.05\right)$. The results of behavioral assessment demonstrated that transfection of RNAi-LV rather than NC-LV could significantly attenuate the development of morphine tolerance (Figure 9D, $p<0.01$ ). Taken together, these results suggest that activation of neuronal ATF6 in spinal cord might be involved in the development of morphine tolerance.

\section{DISCUSSION}

Our study demonstrated the potential role of ER stress in spinal cord in the development of morphine tolerance. BiP, a marker of ER stress and standard indicator of UPR initiation, was up-regulated by chronic morphine treatment in spinal neurons. Three UPR pathways, including IRE1/XBP1 cascade, PERK/eIF2 $\alpha$ cascade, and ATF6, were activated in different neuronal cells in spinal cord during the development of morphine tolerance. Importantly, inhibiting IRE1, PERK, or ATF6 could block UPR pathways and consequently attenuate the development of morphine tolerance, respectively. Taken together, our results provide the direct evidences that ER stress in spinal dorsal horn might be involved in the development of morphine tolerance.

Several mechanisms have been reported to underlie the development of morphine tolerance, including activation of cAMP pathway (Nestler and Aghajanian, 1997), NMDA receptors, CGRP receptors and neuronal GRs (Trujillo and Akil, 1991; Menard et al., 1996; Powell et al., 2000; Lim et al., 2005a,b), 
A

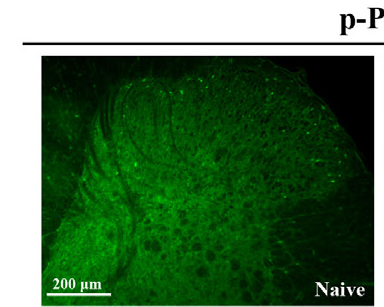

p-PERK
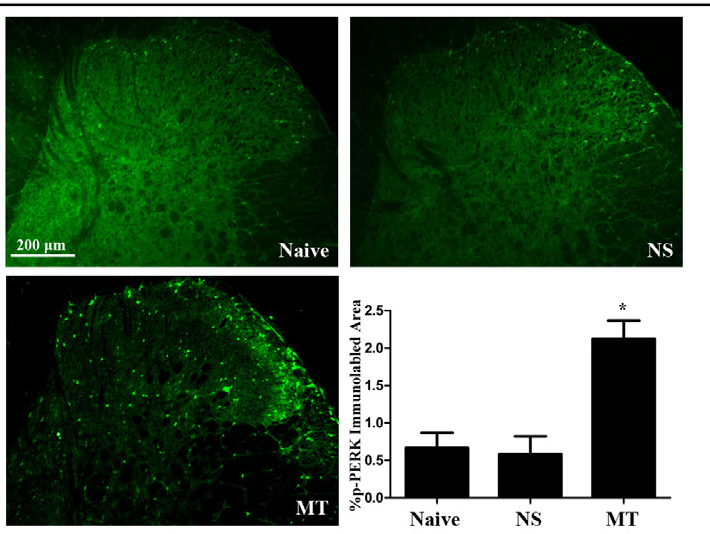

MT

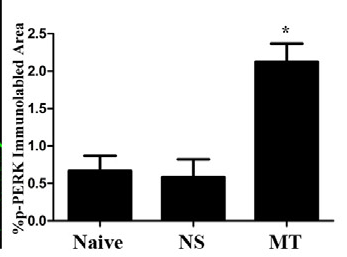

B
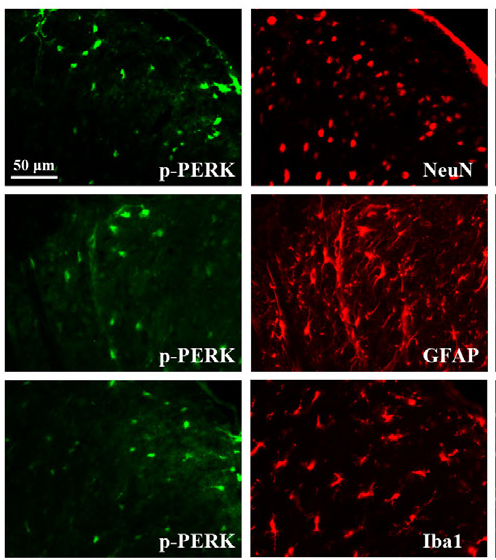
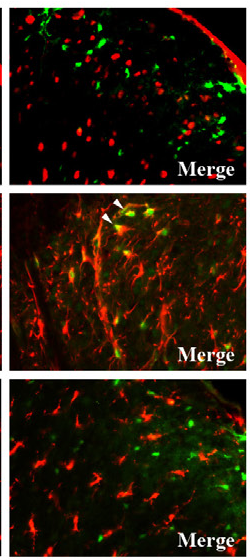

C
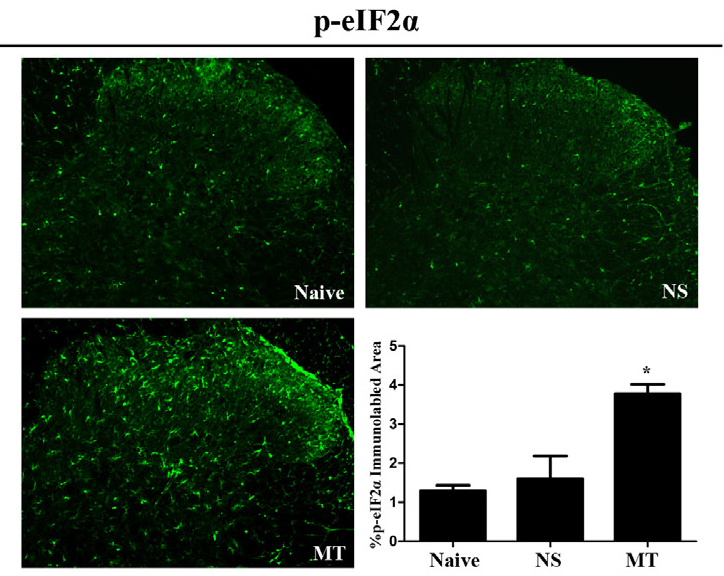

D
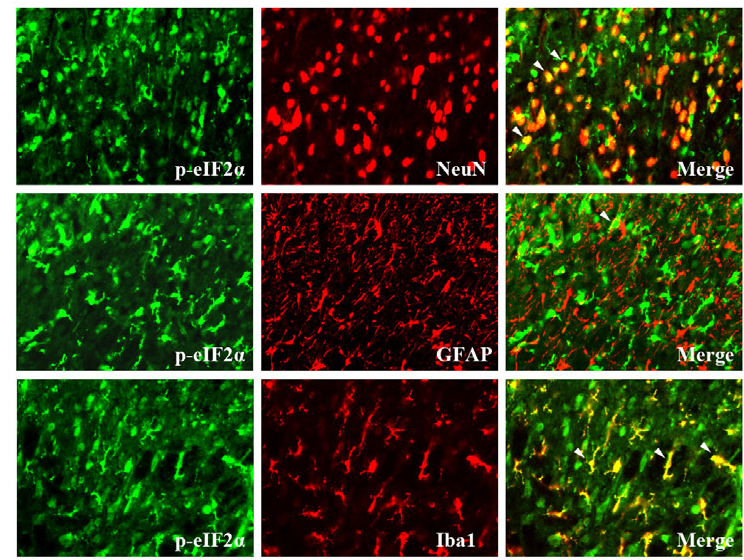

FIGURE 6 | Distributions and cellular localizations of p-PERK and p-elF2 $\alpha$ in spinal cord. (A,C) Immunostaining of p-PERK and p-elF2 $\alpha$ in spinal dorsal horn. P-PERK and p-elF2 $\alpha$ were extensively expressed in spinal dorsal horn. The expressions of p-PERK and p-elF2 $\alpha$ were significantly increased in morphine-tolerant rats compared with those in naïve rats, respectively. ( ${ }^{*} p<0.05$ compared with naive rats, $n=3$ in each group, scale bar: $200 \mu m$ ). (B,D) Double immunostaining of p-PERK and p-elF2 $\alpha$ and cell-specific markers in morphine-tolerant rats. The p-PERK was co-localized with GFAP (indicated by arrows), not with NeuN and Iba1. And p-elF2 $\alpha$ was mainly colocalized with NeuN and lba1 (indicated by arrows), and a minority with GFAP. Scale bar: $50 \mu m$. PERK, protein kinase RNA-like ER kinase; p-PERK, phosphorylation of PERK; elF2 $\alpha$, eukaryotic initiation factor 2 subunit alpha; p-elF2 $\alpha$, phosphorylation of elF2 $\alpha$; NS, normal saline; MT, morphine tolerance.

PKA, PKC, and MAPK (Mao et al., 1994; Cui et al., 2006; Chen et al., 2008; Wang et al., 2009). Recently, a unique phenomenon of morphine was discovered that morphine could alter the balance of excitatory and inhibitory synapses in hippocampus through a pathway involving the generation of reactive oxygen species and subsequent activation of ER stress which eventually initiate autophagy (Cai et al., 2016). ER is a highly dynamic organelle with multiple functions including protein folding and secretion, calcium homeostasis, and lipid biosynthesis (Hawes et al., 2015; Westrate et al., 2015). The physiological functions of ER stress is to induce the activation of UPR and clear unfolded/misfolded proteins and restore ER homeostasis (Rivas et al., 2015; Grootjans et al., 2016). However, excessive or unresolved ER stress inevitably lead to the activation of apoptotic pathways and cell death (Hetz, 2012). Previous studies have shown that ER stress and subsequent UPR were involved in obesity (Ozcan et al., 2004; Hotamisligil, 2005), heart disease (Yamaguchi et al., 2003), ischemia/reperfusion injury (Kumar et al., 2001), diabetes (Guerrero-Hernandez et al., 2014; Engin, 2016), atherosclerosis (Ivanova and Orekhov, 2016; Dong et al., 2017), cancer (Verfaillie et al., 2013; Clarke et al., 2014), Alzheimer's disease and Parkinson's disease (Dawson and Dawson, 2003; Li et al., 2015), as well as various types of chronic pain, including DPN (O’Brien et al., 2014), orofacial inflammatory pain (Yang E.S. et al., 2014), neuropathic pain (Inceoglu et al., 2015; Zhang et al., 2015). BiP is a central player in ER homeostasis. Under physiological conditions, $\mathrm{BiP}$ is associated with the ER stress sensors IRE1, PERK, and ATF6. When ER stress occurs, BiP dissociates from ER stress sensors and binds to misfolded proteins, resulting in the activation of three branches of UPR (O'Brien et al., 2014). Dobashi et al. (2010) reported that $\mathrm{BiP}$, as an ER chaperone, could modulate the development of morphine antinociceptive tolerance. They tested the thermal antinociceptive effect of morphine in heterozygous mutant $\mathrm{BiP}$ mice and found that the mutant BiP mice showed less morphine tolerance. Furthermore, 


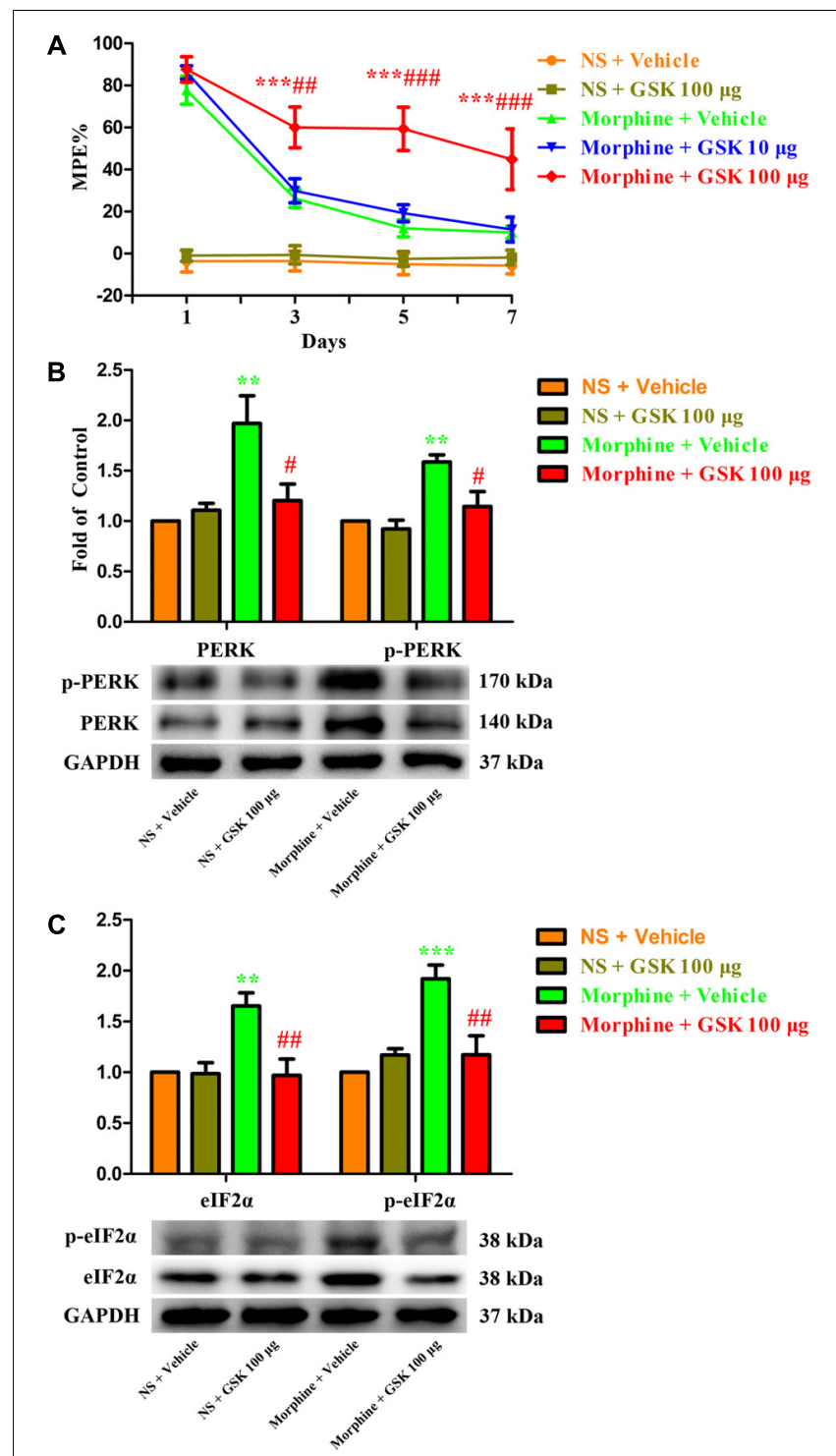

FIGURE 7 | Effect of PERK inhibitor on the development of morphine tolerance. Specific PERK inhibitor GSK2606414 (10 $\mu \mathrm{g}$ or $100 \mu \mathrm{g})$ was intrathecal injected 30 min before morphine treatment for 7 days, respectively. (A) Pretreatment with $100 \mu \mathrm{g}$ GSK2606414 could attenuate the development of morphine tolerance. $\left(^{* * *} p<0.001\right.$ compared with morphine + vehicle group; ${ }^{\#} p<0.01$, \#\#\# $p 0.001$ compared with morphine + GSK $10 \mu \mathrm{g}$ group, $n=6$ in each group). (B,C) Pretreatment with $100 \mu \mathrm{g}$ GSK2606414 could inhibit the up-regulation of PERK, p-PERK (B), elF2 $\alpha$ and p-elF2 $\alpha$ (C) induced by chronic morphine treatment measured by western blots. $\left({ }^{* *} p<0.01,{ }^{* * *} p<0.001\right.$ compared with NS + vehicle group; ${ }^{*} p<0.05$, \#\# $p<0.01$ compared with morphine + vehicle group, $n=6$ in each group). \%MPE, percentage of maximal possible antinociceptive effect; GSK, GSK2606414; PERK, protein kinase RNA-like ER kinase; p-PERK, phosphorylation of PERK; elF2 $\alpha$, eukaryotic initiation factor 2 subunit alpha; p-elF2 $\alpha$, phosphorylation of elF2 $\alpha$; NS, normal saline.

a chemical chaperone called Tauroursodeoxycholic acid which can improve ER protein folding capacity could promote the antinociceptive effect of morphine in wild-type mice, indicating that ER chaperone BiP plays a crucial role in morphine tolerance.
In the present study, we provided the first evidence that the expression of BiP was up-regulated in neurons in spinal dorsal horn after chronic morphine treatment. This indicates that ER stress response and the occurrence of active UPR in spinal cord are closely related to the development of morphine tolerance.

Endoplasmic reticulum stress has been implicated to participate in the mechanism of neuropathic pain (Inceoglu et al., 2015; Zhang et al., 2015), inflammatory pain (Yang E.S. et al., 2014) and SCI in the spinal level (Ohri et al., 2011, 2013). In a rat model of L5 spinal nerve ligation (SNL)-induced neuropathic pain, the ER stress and UPR pathways were significantly activated (Zhang et al., 2015). Moreover, intrathecal administration of ATF6 siRNA attenuated neuropathic pain and BIP expression in the spinal cord. In another study, the activation of ER stress was detected in the peripheral nervous system of diabetic nephropathy rats (Inceoglu et al., 2015). Interestingly, chemical inducers of ER stress could lead to pain behavior which can be reversed by a chemical chaperone. An early activation of UPR pathways could be detected after SCI (Ohri et al., 2011, 2013). Moreover, SCI could lead to the up-regulation of several key ER stress response genes (e.g., ATF4, GADD34, GRP78, and CHOP) at the injury epicenter. Additionally, Genetic and pharmacological modulation of ER stress response via CHOP or PERK/eIF2 $\alpha$ could improve the functional recovery after SCI. These studies provided solid evidence that ER stress play a pivotal role in chronic pain. In this study, we found the increased expressions of both spliced and XBP1u, which are related to IRE1 branch, PERK/eIF2 $\alpha$ and their active forms, as well as ATF6 in spinal cord during the development of morphine tolerance. And pharmacological or genetic blockade of three UPR pathways could attenuate the development of morphine tolerance. So far, only a few studies have reported the effect of opioid on ER stress. In vitro study suggested that morphine could protect primary cultured astrocytes from glutamate-induced apoptosis via reducing $\mathrm{Ca}^{2+}$ overload and ER stress pathways (Zhang et al., 2016). Naloxone, an opioid receptor antagonist, could upregulate gene expression of ER chaperones in PC12 cells, including BiP, calnexin, ER protein 29 and protein disulfide isomerase, and ER stress sensors, including ATF6, IRE1, and PERK. In addition, naloxone could also induce typical ER stress phenomena, including ART6 proteolytic cleavage, eIF2 $\alpha$ phosphorylation and XBP1 mRNA splicing (Seo et al., 2014). Jian et al. (2014) found that the retrieval of morphine memory was associated with the dephosphorylation of eIF $2 \alpha$ expression in basolateral amygdala. Our results confirm the involvement of ER stress including three sensors and related signaling pathways in the development of morphine tolerance. Although blocking either one of three UPR branches could partially prevent the decline of morphine analgesic effect after repeated administration, weight of the pathways in the mechanism of morphine tolerance remains unknown which is hard to determinate. We hypothesized that three branches of UPR may share synergistic effect in the development of morphine tolerance. Therefore, further research is required to explore the complex roles of ER stress in morphine tolerance. 
A

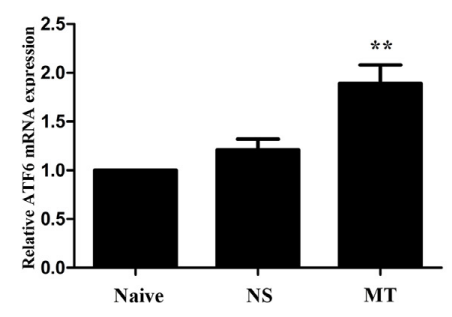

C
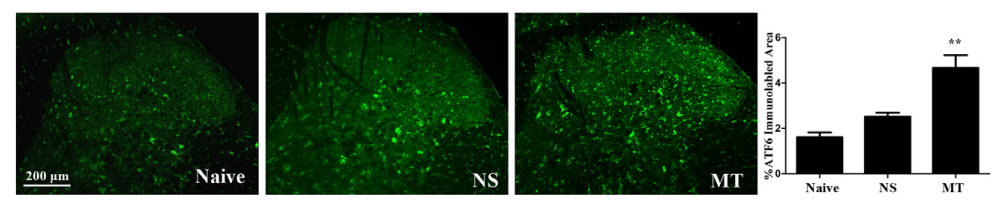

D
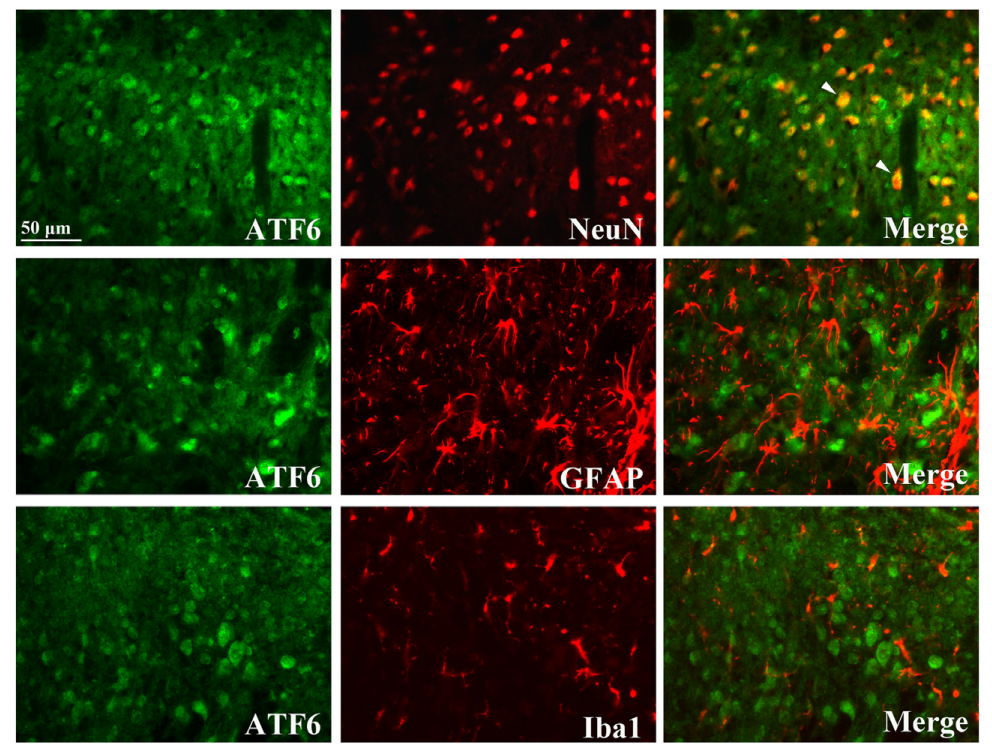

E
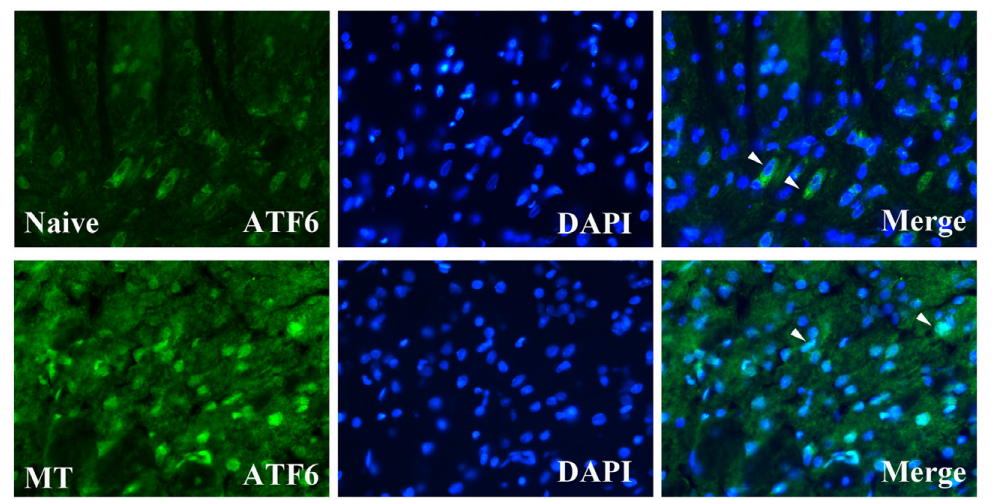

FIGURE 8 | Expression of ATF6 in spinal cord. (A,B) The expression of ATF6 were significantly increased in morphine-tolerant rats measured by real-time PCR and western blots, respectively. $\left(^{* *} p<0.01,{ }^{* * *} p<0.001\right.$ compared with naive rats, Real-time PCR: $n=4$ in each group; western blots: $n=6$ in each group).

(C) Immunostaining of ATF6 in spinal dorsal horn. ATF6 was extensively expressed in spinal dorsal horn. The immunoreactivity of ATF6 was significantly increased in morphine-tolerant rats. ( ${ }^{* *} p<0.01$ compared with naive rats, $n=3$ in each group, scale bar: $\left.200 \mu \mathrm{m}\right)$. (D) Double immunostaining of ATF6 and cell-specific markers in morphine-tolerant rats. ATF6 was co-localized with NeuN (indicated by arrows), not with GFAP or lba1 in spinal dorsal horn. Scale bar: $50 \mu \mathrm{m}$. (E) Chronic morphine administration results in ATF6 translocation to the nucleus. ATF6 was only expressed in cytoplasm in naive rats (indicated by arrows), while ATF6 was expressed in cytoplasm and cell nucleus in morphine-tolerant rats (indicated by arrows). Scale bar: 50 m. ATF6, activating transcription factor 6; NS, normal saline; MT, morphine tolerance. 
A
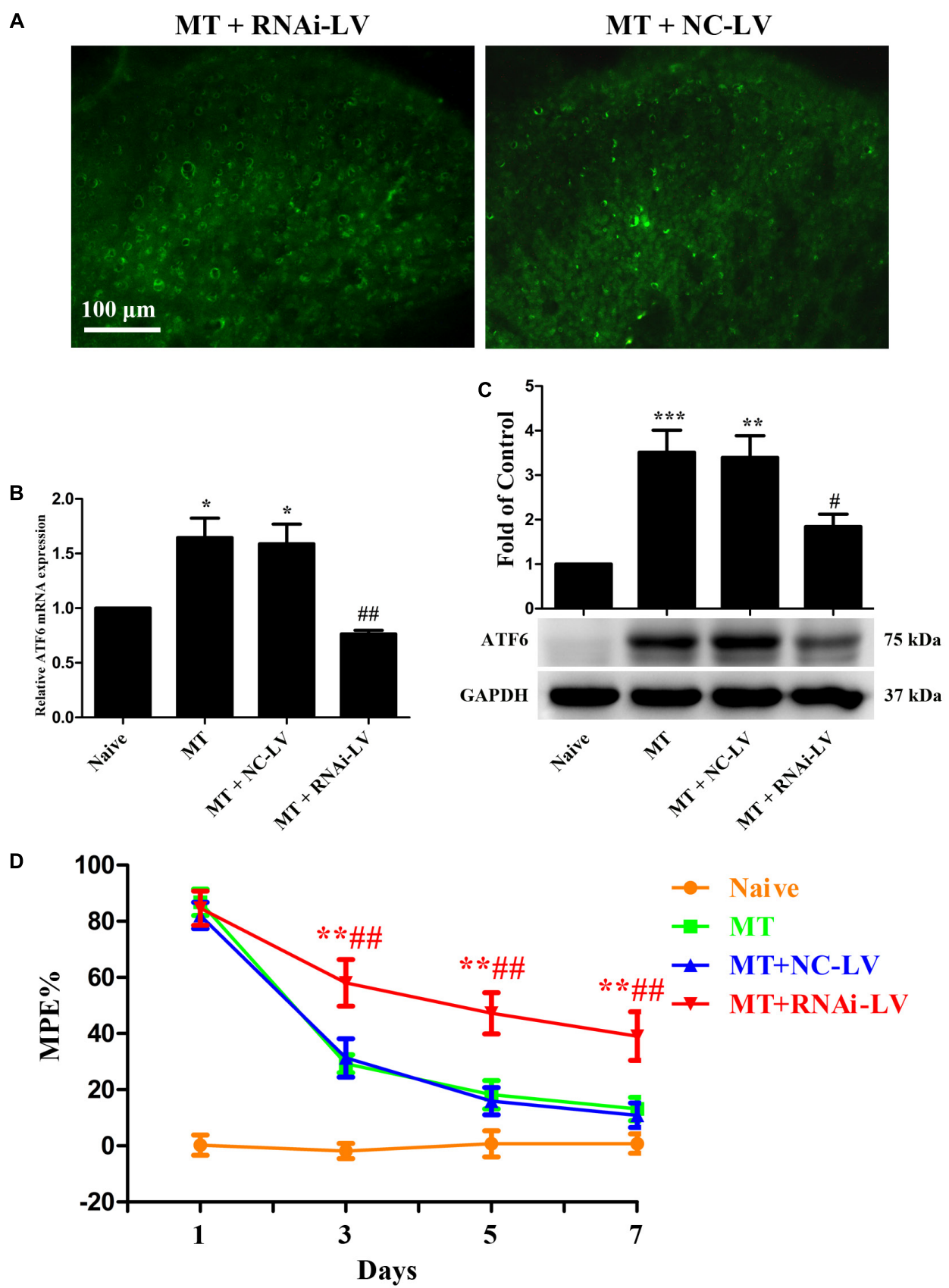

FIGURE 9 | Effect of ATF6 RNAi-Lentivirus on the development of morphine tolerance. (A) Detection of lentivirus transfection in spinal cord. RNAi-LV or NC-LV was injected into lumbar spinal cord 3 days before intrathecal catheterization, respectively. The expression of enhanced green fluorescent protein in lentiviral vectors was detected in spinal dorsal horn $n=3$ in each group. Scale bar: $100 \mu \mathrm{m}$. (B,C) RNAi-LV could effectively downregulate the expression of ATF6 induced by chronic morphine treatment measured by real-time PCR and western blots, respectively. ${ }^{*} p<0.05,{ }^{* *} p<0.01,{ }^{* * *} p<0.001$ compared with naive rats, ${ }^{*} p<0.05$, ${ }^{\# \#} p<0.01$ compared with MT+NC-LV group, Real-time PCR: $n=4$ in each group; western blots: $n=6$ in each group). (D) Pretreatment with RNAi-LV could attenuate the development of morphine tolerance. (** $p<0.01$ compared with MT group; $\# \#<0.01$ compared with MT+NC-LV group, $n=6-8$ in each group). NS, normal saline; MT, morphine tolerance; ATF6, activating transcription factor 6; \%MPE, percentage of maximal possible antinociceptive effect.

Our previous studies have demonstrated the important role and complexity of neuron-glia interaction in the mechanism of morphine tolerance (Peng et al., 2017; Wang et al., 2017). In the present study, expressions of IRE1 and XBP1 were found to be increased mainly in neurons and astrocytes in spinal dorsal horn, while ATF6 expression was increased mainly in spinal neurons in morphine-tolerant rats. In supraspinal mechanisms of CNS disorders, the increased expressions of IRE1, XBP1, and ATF6 could be observed in neurons and astrocytes in different brain regions, which indicate the interaction between neurons 
and glia (Zhang et al., 2012; Yang W. et al., 2014; Wen et al., 2016; Duran-Aniotz et al., 2017). Our results imply that neurons might be specifically responsible for pain signaling in cooperation with ER stress response. Additionally, in our study, p-PERK was found to be activated mainly in astrocytes of the spinal dorsal horn in morphine-tolerant rats, while p-eIF $2 \alpha$ mainly in neurons and microglia, and only a minority of p-eIF $2 \alpha$ in astrocytes. Our results indicated that PERK/eIF $2 \alpha$ cascade might play a fundamental role in neuron-glia and astrocyte-microglia interactions during the development of morphine tolerance. Previous study reported that PERK-eIF2 $\alpha$ pathway was activated in astrocytes of the spinal dorsal horn in SNL model (Zhang et al., 2015). Phosphorylated eIF $2 \alpha$ could be induced in spinal interneurons in a rabbit spinal cord ischemia model (Yamauchi et al., 2007). Therefore, PERK-eIF2 $\alpha$ pathway may take part in the different pathophysiological processes through different patterns. We speculate that there may be a different function of PERK-eIF $2 \alpha$ pathway in morphine tolerance compared to IRE1-XBP1 and ATF6 pathways. Thus, further research about PERK-eIF2 $\alpha$ pathway in morphine tolerance is worthy to do.

In summary, our study provides the first direct evidences that ER stress might be a significant driver of the development of

\section{REFERENCES}

Bertolotti, A., Zhang, Y., Hendershot, L. M., Harding, H. P., and Ron, D. (2000). Dynamic interaction of $\mathrm{BiP}$ and ER stress transducers in the unfolded-protein response. Nat. Cell Biol. 2, 326-332. doi: 10.1038/35014014

Bettigole, S. E., and Glimcher, L. H. (2015). Endoplasmic reticulum stress in immunity. Annu. Rev. Immunol. 33, 107-138. doi: 10.1146/annurev-immunol032414-112116

Cai, Y., Yang, L., Hu, G., Chen, X., Niu, F., Yuan, L., et al. (2016). Regulation of morphine-induced synaptic alterations: role of oxidative stress, ER stress, and autophagy. J. Cell Biol. 215, 245-258. doi: 10.1083/jcb.201605065

Chen, Y., Geis, C., and Sommer, C. (2008). Activation of TRPV1 contributes to morphine tolerance: involvement of the mitogen-activated protein kinase signaling pathway. J. Neurosci. 28, 5836-5845. doi: 10.1523/JNEUROSCI.417007.2008

Clarke, H. J., Chambers, J. E., Liniker, E., and Marciniak, S. J. (2014). Endoplasmic reticulum stress in malignancy. Cancer Cell 25, 563-573. doi: 10.1016/j.ccr.2014. 03.015

Cui, Y., Chen, Y., Zhi, J. L., Guo, R. X., Feng, J. Q., and Chen, P. X. (2006). Activation of $\mathrm{p} 38$ mitogen-activated protein kinase in spinal microglia mediates morphine antinociceptive tolerance. Brain Res. 1069, 235-243. doi: 10.1016/j. brainres.2005.11.066

Cui, Y., Liao, X. X., Liu, W., Guo, R. X., Wu, Z. Z., Zhao, C. M., et al. (2008). A novel role of minocycline: attenuating morphine antinociceptive tolerance by inhibition of p38 MAPK in the activated spinal microglia. Brain Behav. Immun. 22, 114-123. doi: 10.1016/j.bbi.2007.07.014

Dawson, T. M., and Dawson, V. L. (2003). Rare genetic mutations shed light on the pathogenesis of Parkinson disease. J. Clin. Invest. 111, 145-151. doi: 10.1172/JCI200317575

Dobashi, T., Tanabe, S., Jin, H., Mimura, N., Yamamoto, T., Nishino, T., et al. (2010). BiP, an endoplasmic reticulum chaperone, modulates the development of morphine antinociceptive tolerance. J. Cell. Mol. Med. 14, 2816-2826. doi: 10.1111/j.1582-4934.2009.00932.x

Dong, Y., Fernandes, C., Liu, Y., Wu, Y., Wu, H., Brophy, M. L., et al. (2017). Role of endoplasmic reticulum stress signalling in diabetic endothelial dysfunction and atherosclerosis. Diab. Vasc. Dis. Res. 14, 14-23. doi: 10.1177/1479164116666762

Doyle, K. M., Kennedy, D., Gorman, A. M., Gupta, S., Healy, S. J., and Samali, A. (2011). Unfolded proteins and endoplasmic reticulum stress in neurodegenerative disorders. J. Cell. Mol. Med. 15, 2025-2039. doi: 10.1111/j. 1582-4934.2011.01374.x morphine tolerance in spinal cord. These findings implicate a potential therapeutic strategy to restore the antinociceptive effect of morphine by inhibiting ER stress sensors IRE1, PERK, and ATF6, which may contribute to expanding the morphine usage in clinical practice.

\section{AUTHOR CONTRIBUTIONS}

FG supervised the entire project, designed the research, and wrote the paper. DL wrote and edited the manuscript and conceived and designed the ideas. YZ, YP, PS, ZL, and QX interpreted and analyzed the data. YT, XT, HY, ZW, and WM made substantial, direct, and intellectual contribution to the work. All authors read and approved the final manuscript.

\section{FUNDING}

This work was supported by grants from the National Natural Science Foundation of China (No. 81771191).

Duran-Aniotz, C., Cornejo, V. H., Espinoza, S., Ardiles, A. O., Medinas, D. B., Salazar, C., et al. (2017). IRE1 signaling exacerbates Alzheimer's disease pathogenesis. Acta Neuropathol. 134, 489-506. doi: 10.1007/s00401-017-1694-x

Engin, F. (2016). ER stress and development of type 1 diabetes. J. Investig. Med. 64, 2-6.

Fu, Q., Shi, D., Zhou, Y., Zheng, H., Xiang, H., Tian, X., et al. (2016). MHCI promotes apoptosis of GABAergic interneurons in the spinal dorsal horn and contributes to cancer induced bone pain. Exp. Neurol. 286, 12-20. doi: 10.1016/j.expneurol.2016.09.002

Grootjans, J., Kaser, A., Kaufman, R. J., and Blumberg, R. S. (2016). The unfolded protein response in immunity and inflammation. Nat. Rev. Immunol. 16, 469-484. doi: 10.1038/nri.2016.62

Guerrero-Hernandez, A., Leon-Aparicio, D., Chavez-Reyes, J., Olivares-Reyes, J. A., and Dejesus, S. (2014). Endoplasmic reticulum stress in insulin resistance and diabetes. Cell Calcium 56, 311-322. doi: 10.1016/j.ceca.2014.08.006

Guo, G., and Gao, F. (2015). CXCR3: latest evidence for the involvement of chemokine signaling in bone cancer pain. Exp. Neurol. 265, 176-179. doi: 10.1016/j.expneurol.2015.02.003

Guo, G., Peng, Y., Xiong, B., Liu, D., Bu, H., Tian, X., et al. (2016). Involvement of chemokine CXCL11 in the development of morphine tolerance in rats with cancer-induced bone pain. J. Neurochem. 141, 553-564. doi: 10.1111/jnc.13919

Guo, G., Peng, Y., Xiong, B., Liu, D., Bu, H., Tian, X., et al. (2017). Involvement of chemokine CXCL11 in the development of morphine tolerance in rats with cancer-induced bone pain. J. Neurochem. 141, 553-564. doi: 10.1111/jnc.13919

Hawes, C., Kiviniemi, P., and Kriechbaumer, V. (2015). The endoplasmic reticulum: a dynamic and well-connected organelle. J. Integr Plant Biol. 57, 50-62. doi: 10.1111/jipb.12297

Hetz, C. (2012). The unfolded protein response: controlling cell fate decisions under ER stress and beyond. Nat. Rev. Mol. Cell Biol. 13, 89-102. doi: 10.1038/ nrm 3270

Hotamisligil, G. S. (2005). Role of endoplasmic reticulum stress and c-Jun NH2terminal kinase pathways in inflammation and origin of obesity and diabetes. Diabetes 54(Suppl. 2), S73-S78. doi: 10.2337/diabetes.54.suppl_2.S73

Inceoglu, B., Bettaieb, A., Trindade Da Silva, C. A., Lee, K. S., Haj, F. G., and Hammock, B. D. (2015). Endoplasmic reticulum stress in the peripheral nervous system is a significant driver of neuropathic pain. Proc. Natl. Acad. Sci. U.S.A. 112, 9082-9087. doi: 10.1073/pnas.1510137112

Ivanova, E. A., and Orekhov, A. N. (2016). The role of endoplasmic reticulum stress and unfolded protein response in atherosclerosis. Int. J. Mol. Sci. 17:193. doi: $10.3390 /$ ijms 17020193 
Jian, M., Luo, Y. X., Xue, Y. X., Han, Y., Shi, H. S., Liu, J. F., et al. (2014). eIF2alpha dephosphorylation in basolateral amygdala mediates reconsolidation of drug memory. J. Neurosci. 34, 10010-10021. doi: 10.1523/JNEUROSCI.0934- 14.2014

King, T., Ossipov, M. H., Vanderah, T. W., Porreca, F., and Lai, J. (2005). Is paradoxical pain induced by sustained opioid exposure an underlying mechanism of opioid antinociceptive tolerance? Neurosignals 14, 194-205. doi: $10.1159 / 000087658$

Kumar, R., Azam, S., Sullivan, J. M., Owen, C., Cavener, D. R., Zhang, P., et al. (2001). Brain ischemia and reperfusion activates the eukaryotic initiation factor 2alpha kinase. PERK. J. Neurochem. 77, 1418-1421. doi: 10.1046/j.1471-4159. 2001.00387.x

Lauro, F., Giancotti, L. A., Ilari, S., Dagostino, C., Gliozzi, M., Morabito, C., et al. (2016). Inhibition of spinal oxidative stress by bergamot polyphenolic fraction attenuates the development of morphine induced tolerance and hyperalgesia in mice. PLoS One 11:e0156039. doi: 10.1371/journal.pone.015 6039

Li, J. Q., Yu, J. T., Jiang, T., and Tan, L. (2015). Endoplasmic reticulum dysfunction in Alzheimer's disease. Mol. Neurobiol. 51, 383-395. doi: 10.1007/s12035-0148695-8

Lim, G., Wang, S., Zeng, Q., Sung, B., and Mao, J. (2005a). Evidence for a longterm influence on morphine tolerance after previous morphine exposure: role of neuronal glucocorticoid receptors. Pain 114, 81-92.

Lim, G., Wang, S., Zeng, Q., Sung, B., and Mao, J. (2005b). Spinal glucocorticoid receptors contribute to the development of morphine tolerance in rats. Anesthesiology 102, 832-837. doi: 10.1097/00000542-20050400000020

Liu, M. Q., Chen, Z., and Chen, L. X. (2016). Endoplasmic reticulum stress: a novel mechanism and therapeutic target for cardiovascular diseases. Acta Pharmacol. Sin. 37, 425-443. doi: 10.1038/aps.2015.145

Mao, J., Price, D. D., and Mayer, D. J. (1994). Thermal hyperalgesia in association with the development of morphine tolerance in rats: roles of excitatory amino acid receptors and protein kinase C. J. Neurosci. 14, 2301-2312.

Martini, L., and Whistler, J. L. (2007). The role of mu opioid receptor desensitization and endocytosis in morphine tolerance and dependence. Curr. Opin. Neurobiol. 17, 556-564. doi: 10.1016/j.conb.2007.10.004

Menard, D. P., Van Rossum, D., Kar, S., St Pierre, S., Sutak, M., Jhamandas, K., et al. (1996). A calcitonin gene-related peptide receptor antagonist prevents the development of tolerance to spinal morphine analgesia. J. Neurosci. 16, 2342-2351.

Mori, K. (2015). The unfolded protein response: the dawn of a new field. Proc. Jpn. Acad. Ser. B Phys. Biol. Sci. 91, 469-480. doi: 10.2183/pjab.91.469

Narita, M., Suzuki, M., Narita, M., Niikura, K., Nakamura, A., Miyatake, M., et al. (2006). mu-Opioid receptor internalization-dependent and -independent mechanisms of the development of tolerance to mu-opioid receptor agonists: comparison between etorphine and morphine. Neuroscience 138, 609-619. doi: 10.1016/j.neuroscience.2005.11.046

Nestler, E. J., and Aghajanian, G. K. (1997). Molecular and cellular basis of addiction. Science 278, 58-63. doi: 10.1126/science.278.5335.58

O'Brien, P. D., Hinder, L. M., Sakowski, S. A., and Feldman, E. L. (2014). ER stress in diabetic peripheral neuropathy: a new therapeutic target. Antioxid. Redox Signal. 21, 621-633. doi: 10.1089/ars.2013.5807

Ohri, S. S., Hetman, M., and Whittemore, S. R. (2013). Restoring endoplasmic reticulum homeostasis improves functional recovery after spinal cord injury. Neurobiol. Dis. 58, 29-37. doi: 10.1016/j.nbd.2013.04.021

Ohri, S. S., Maddie, M. A., Zhao, Y., Qiu, M. S., Hetman, M., and Whittemore, S. R. (2011). Attenuating the endoplasmic reticulum stress response improves functional recovery after spinal cord injury. Glia 59, 1489-1502. doi: 10.1002/ glia. 21191

Ozcan, U., Cao, Q., Yilmaz, E., Lee, A. H., Iwakoshi, N. N., Ozdelen, E., et al. (2004). Endoplasmic reticulum stress links obesity, insulin action, and type 2 diabetes. Science 306, 457-461. doi: 10.1126/science.1103160

Pasternak, G. W., and Pan, Y. X. (2011). Mix and match: heterodimers and opioid tolerance. Neuron 69, 6-8. doi: 10.1016/j.neuron.2010.12.030

Penas, C., Guzman, M. S., Verdu, E., Fores, J., Navarro, X., and Casas, C. (2007). Spinal cord injury induces endoplasmic reticulum stress with different cell-type dependent response. J. Neurochem. 102, 1242-1255. doi: 10.1111/j.1471-4159. 2007.04671.x
Peng, Y., Guo, G., Shu, B., Liu, D., Su, P., Zhang, X., et al. (2017). Spinal CX3CL1/CX3CR1 may not directly participate in the development of morphine tolerance in rats. Neurochem. Res. 42, 3254-3267. doi: 10.1007/s11064-0172364-z

Piperi, C., Adamopoulos, C., Dalagiorgou, G., Diamanti-Kandarakis, E., and Papavassiliou, A. G. (2012). Crosstalk between advanced glycation and endoplasmic reticulum stress: emerging therapeutic targeting for metabolic diseases. J. Clin. Endocrinol. Metab. 97, 2231-2242. doi: 10.1210/jc.20113408

Powell, K. J., Ma, W., Sutak, M., Doods, H., Quirion, R., and Jhamandas, K. (2000). Blockade and reversal of spinal morphine tolerance by peptide and non-peptide calcitonin gene-related peptide receptor antagonists. Br. J. Pharmacol. 131, 875-884. doi: 10.1038/sj.bjp.0703655

Rivas, A., Vidal, R. L., and Hetz, C. (2015). Targeting the unfolded protein response for disease intervention. Expert Opin. Ther. Targets 19, 1203-1218. doi: 10.1517/ 14728222.2015.1053869

Ron, D., and Walter, P. (2007). Signal integration in the endoplasmic reticulum unfolded protein response. Nat. Rev. Mol. Cell Biol. 8, 519-529. doi: 10.1038/ nrm2199

Seo, H. Y., Kim, M. K., Min, A. K., Kim, H. S., Ryu, S. Y., Kim, N. K., et al. (2010). Endoplasmic reticulum stress-induced activation of activating transcription factor 6 decreases cAMP-stimulated hepatic gluconeogenesis via inhibition of CREB. Endocrinology 151, 561-568. doi: 10.1210/en.20090641

Seo, H. Y., Kim, Y. D., Lee, K. M., Min, A. K., Kim, M. K., Kim, H. S., et al. (2008). Endoplasmic reticulum stress-induced activation of activating transcription factor 6 decreases insulin gene expression via up-regulation of orphan nuclear receptor small heterodimer partner. Endocrinology 149, 3832-3841. doi: 10.1210/en.2008-0015

Seo, S., Kwon, Y. S., Yu, K., Kim, S. W., Kwon, O. Y., Kang, K. H., et al. (2014). Naloxone induces endoplasmic reticulum stress in PC12 cells. Mol. Med. Rep. 9, 1395-1399. doi: 10.3892/mmr.2014.1935

Szentirmay, A. K., Kiraly, K. P., Lenkey, N., Lacko, E., Al-Khrasani, M., Friedmann, T., et al. (2013). Spinal interaction between the highly selective mu agonist DAMGO and several delta opioid receptor ligands in naive and morphine-tolerant mice. Brain Res. Bull. 90, 66-71. doi: 10.1016/j.brainresbull. 2012.09.006

Trujillo, K. A., and Akil, H. (1991). Inhibition of morphine tolerance and dependence by the NMDA receptor antagonist MK-801. Science 251, 85-87. doi: $10.1126 /$ science. 1824728

Verfaillie, T., Garg, A. D., and Agostinis, P. (2013). Targeting ER stress induced apoptosis and inflammation in cancer. Cancer Lett. 332, 249-264. doi: 10.1016/ j.canlet.2010.07.016

Wang, W., Peng, Y., Yang, H., Bu, H., Guo, G., Liu, D., et al. (2017). Potential role of CXCL10/CXCR3 signaling in the development of morphine tolerance in periaqueductal gray. Neuropeptides 65, 120-127. doi: 10.1016/j.npep.2017. 07.004

Wang, W. A., Groenendyk, J., and Michalak, M. (2014). Endoplasmic reticulum stress associated responses in cancer. Biochim. Biophys. Acta 1843, 2143-2149. doi: 10.1016/j.bbamcr.2014.01.012

Wang, Z., Ma, W., Chabot, J. G., and Quirion, R. (2009). Cell-type specific activation of p38 and ERK mediates calcitonin gene-related peptide involvement in tolerance to morphine-induced analgesia. FASEB J. 23, 2576-2586. doi: 10.1096/fj.08-128348

Wen, L., Han, F., Shi, Y., and Li, X. (2016). Role of the endoplasmic reticulum pathway in the medial prefrontal cortex in post-traumatic stress disorder model rats. J. Mol. Neurosci. 59, 471-482. doi: 10.1007/s12031-016-0755-2

Westrate, L. M., Lee, J. E., Prinz, W. A., and Voeltz, G. K. (2015). Form follows function: the importance of endoplasmic reticulum shape. Annu. Rev. Biochem. 84, 791-811. doi: 10.1146/annurev-biochem-072711-163501

Williams, J. T., Ingram, S. L., Henderson, G., Chavkin, C., Von Zastrow, M., Schulz, S., et al. (2013). Regulation of mu-opioid receptors: desensitization, phosphorylation, internalization, and tolerance. Pharmacol. Rev. 65, 223-254. doi: 10.1124/pr.112.005942

Xu, C., Bailly-Maitre, B., and Reed, J. C. (2005). Endoplasmic reticulum stress: cell life and death decisions. J. Clin. Invest. 115, 2656-2664. doi: 10.1172/JCI26373

Yamaguchi, O., Higuchi, Y., Hirotani, S., Kashiwase, K., Nakayama, H., Hikoso, S., et al. (2003). Targeted deletion of apoptosis signal-regulating kinase 1 attenuates 
left ventricular remodeling. Proc. Natl. Acad. Sci. U.S.A. 100, 15883-15888. doi: $10.1073 /$ pnas.2136717100

Yamauchi, T., Sakurai, M., Abe, K., Matsumiya, G., and Sawa, Y. (2007). Impact of the endoplasmic reticulum stress response in spinal cord after transient ischemia. Brain Res. 1169, 24-33. doi: 10.1016/j.brainres.2007.06.093

Yang, E. S., Bae, J. Y., Kim, T. H., Kim, Y. S., Suk, K., and Bae, Y. C. (2014). Involvement of endoplasmic reticulum stress response in orofacial inflammatory pain. Exp. Neurobiol. 23, 372-380. doi: 10.5607/en.2014.23. 4.372

Yang, W., Shen, Y., Chen, Y., Chen, L., Wang, L., Wang, H., et al. (2014). Mesencephalic astrocyte-derived neurotrophic factor prevents neuron loss via inhibiting ischemia-induced apoptosis. J. Neurol. Sci. 344, 129-138. doi: 10.1016/j.jns.2014.06.042

Ye, D., Bu, H., Guo, G., Shu, B., Wang, W., Guan, X., et al. (2014). Activation of CXCL10/CXCR3 signaling attenuates morphine analgesia: involvement of Gi protein. J. Mol. Neurosci. 53, 571-579. doi: 10.1007/s12031-0130223-1

Zhang, C., Wang, C., Ren, J., Guo, X., and Yun, K. (2016). Morphine protects spinal cord astrocytes from glutamate-induced apoptosis via reducing endoplasmic reticulum stress. Int. J. Mol. Sci. 17:E1523. doi: 10.3390/ijms1710 1523
Zhang, E., Yi, M. H., Shin, N., Baek, H., Kim, S., Kim, E., et al. (2015). Endoplasmic reticulum stress impairment in the spinal dorsal horn of a neuropathic pain model. Sci. Rep. 5:11555. doi: 10.1038/srep11555

Zhang, Y., Liu, W., Ma, C., Geng, J., Li, Y., Li, S., et al. (2012). Endoplasmic reticulum stress contributes to $\mathrm{CRH}$-induced hippocampal neuron apoptosis. Exp. Cell Res. 318, 732-740. doi: 10.1016/j.yexcr.2012.01.006

Zhou, Y. Q., Chen, S. P., Liu, D. Q., Manyande, A., Zhang, W., Yang, S. B., et al. (2017). The role of spinal GABAB receptors in cancer-induced bone pain in rats. J. Pain 18, 933-946. doi: 10.1016/j.jpain.2017.02.438

Conflict of Interest Statement: The authors declare that the research was conducted in the absence of any commercial or financial relationships that could be construed as a potential conflict of interest.

Copyright (C) $2018 \mathrm{Liu}$, Zhou, Peng, Su, Li, Xu, Tu, Tian, Yang, Wu, Mei and Gao. This is an open-access article distributed under the terms of the Creative Commons Attribution License (CC BY). The use, distribution or reproduction in other forums is permitted, provided the original author(s) and the copyright owner are credited and that the original publication in this journal is cited, in accordance with accepted academic practice. No use, distribution or reproduction is permitted which does not comply with these terms. 